\title{
Jet quenching from heavy to light ion collisions
}

\section{B.G. Zakharov}

L.D. Landau Institute for Theoretical Physics, GSP-1, 117940, Kosygina Str.2, 117334 Moscow, Russia

Steklov Mathematical Institute, Russian Academy of Sciences, Gubkin Str. 8, 119991 Moscow, Russia

E-mail: bgz@itp.ac.ru

ABSTRACT: We perform an analysis of jet quenching in heavy and light ion collisions for scenarios without and with quark-gluon plasma formation in $p p$ collisions. We find that the results for these scenarios are very similar, and both of them are in reasonable agreement with data for heavy ion collisions. However, their results become differ significantly for light nuclei. Using the parameters fitted to heavy ion data on the nuclear modification factor $R_{A A}$, we make predictions for 0.2 and $7 \mathrm{TeV} \mathrm{O}+\mathrm{O}$ collisions that can be verified by future experiments at RHIC and the LHC.

KEYwords: Heavy Ion Phenomenology, Jets

ArXiv EPrint: 2105.09350 


\section{Contents}

1 Introduction 1

2 Basic aspects of the jet quenching scheme $\quad 4$

3 Models of the QGP fireballs for $A A$ and $p p$ collisions 8

3.1 The QGP fireball in $A A$ collisions $\quad 9$

$\begin{array}{lll}3.2 & \text { The mQGP fireball parameters for } p p \text { collisions } & 11\end{array}$

4 Numerical results $\quad 13$

4.1 Optimal $\kappa$ from the $\chi^{2}$ fit 13

$\begin{array}{lll}4.2 & \text { Predictions for } R_{p p}, R_{A A} \text { and azimuthal asymmetry } v_{2} & 13\end{array}$

$\begin{array}{lll}4.3 & \text { Jet quenching in } p A \text { collisions } & 21\end{array}$

5 Conclusions $\quad 23$

\section{Introduction}

It is believed that heavy ion collisions at the RHIC and LHC energies produce a deconfined quark-gluon plasma (QGP). Strong suppression of the high- $p_{T}$ particles observed in heavy ion collisions (usually called jet quenching) at RHIC and the LHC is one of the major signals of the QGP formation. This phenomenon is a consequence of parton energy loss in the QGP. For fast partons the energy loss is dominated by the radiative mechanism through induced gluon emission [1-6]. The suppression of particle spectra in $A A$ collisions is quantified by the nuclear modification factor $R_{A A}$. For a given centrality bin $\Delta c$ (which is experimentally determined via charged hadron multiplicities) $R_{A A}$ is defined as the ratio of particle yield in $A A$ collisions to that in $p p$ collisions scaled with the number of binary collisions

$$
R_{A A}=\frac{d^{2} N_{A A} / d p_{T}^{2} d y}{N_{\mathrm{ev}}\left\langle T_{A A}\right\rangle_{\Delta c} d^{2} \sigma_{p p} / d p_{T}^{2} d y},
$$

where $\left\langle T_{A A}\right\rangle_{\Delta c}$ is the nuclear overlap function for centrality class $\Delta c$. The relation between centrality $c$ and the impact parameter $b$ of $A A$ collision is usually defined within the Glauber model. Theoretical calculations of the nuclear suppression are commonly performed assuming that the medium jet modification occurs only in $A A$ collisions, and is absent in $p p$ collisions, i.e., the $p p$ yield in the denominator on the righthand side of (1.1) can be calculated in the standard pQCD approach. In this scenario, with the Glauber initial conditions for the QGP fireball at the proper time $\tau_{0} \sim 0.5 \mathrm{fm}$, one can obtain within pQCD jet quenching models rather good description of the available data on the nuclear modification factor from $\mathrm{RHIC}$ for $\mathrm{Au}+\mathrm{Au}$ collisions and from $\mathrm{LHC}$ for $\mathrm{Pb}+\mathrm{Pb}$ and $\mathrm{Xe}+\mathrm{Xe}$ 
collisions (see, e.g., refs. [7-11]). However, it is possible that the assumption that QGP is not formed in $p p$ collisions is not valid. The observation of the ridge effect in highmultiplicity $p p$ collisions at the LHC [12] (and even in lower-multiplicity $p p$ collisions [13]), suggests that a small size QGP fireball can be created in $p p$ collisions as well, because the hydrodynamic transverse flow seems to be the most natural explanation for the ridge effect. But an alternative interpretation of the ridge phenomenon via the initial state effects [14], without the collective flow in the final state, is not excluded. The formation of the QGP droplets in collisions of small systems is now one of the challenging issues for high energy QCD. Future experiments on light ion collisions at RHIC [15] and at the LHC [16-18] will allow to understand better the role of the collective effects in small systems.

In the case of a mini-QGP (mQGP) formation in $p p$ collisions, even if it occurs only in a part of jet events, the parton shower and the inclusive $p p$ cross section will be affected by the parton energy loss in the mQGP. It is important that the jet events in $p p$ collisions have favorable conditions for QGP formation, as compared to the minimum bias events, since the charged multiplicity density of the soft (underlying-event (UE)) hadrons in the jet events, $d N_{\mathrm{ch}}^{\mathrm{ue}} / d \eta$, turns out to be bigger than the ordinary minimum bias multiplicity density by a factor (we denote it as $K_{\text {ue }}$ ) of $\sim 2-2.5$ [19]. The $K_{\text {ue }}$ grows with momentum of the leading charged jet hadron at $p_{T} \lesssim 3-5 \mathrm{GeV}$, and then flattens out [20-24]. The enhancement of the UE multiplicity may be due to the fact that jet production should be most probable for central $p p$ collisions, and due to the initial state radiation that accompanies hard jet production. For the LHC energies $d N_{\mathrm{ch}}^{\mathrm{ue}} / d \eta$ at $\eta \sim 0$ turns out to be larger than the critical pseudorapidity density $d N_{\mathrm{ch}} / d \eta \sim 6$ for beginning of the regime with formation of the hot QCD matter in $p p$ collisions found in [25] from variation of the average transverse hadron momentum $\left\langle p_{T}\right\rangle$ with $d N_{\mathrm{ch}} / d \eta$, and than a more conservative value $d N_{\mathrm{ch}} / d \eta \sim 9$ obtained in [26]. ${ }^{1}$ The onset of the regime with QGP formation at $d N_{\mathrm{ch}} / d \eta \gtrsim 5-10$ is also supported by the observation of a steep growth of the strange particle production in $p p$ collisions in the range $d N_{\mathrm{ch}} / d \eta \sim 2-8$ [27]. For $p p$ collisions at the RHIC energy $\sqrt{s}=0.2 \mathrm{TeV}$ $d N_{\mathrm{ch}}^{\mathrm{ue}} / d \eta \sim 6$. Thus, one can expect for the UEs in jet production in $p p$ collisions at RHIC energies the thermalization may be incomplete. Of course, the transition to the regime of a thermalized QCD matter may be smooth. It is possible that at the beginning the final state interactions in the parton system are weak and lead only to a small modification of the free-stream evolution [28]. Also, it is clear that, due to event-by-event fluctuations of the entropy/energy production, the QGP can be formed in some part of jet events even for a small average UE multiplicity density at $\sqrt{s}=0.2 \mathrm{TeV}$. Therefore, some medium jet modification in $p p$ collisions may already occur at the RHIC energies.

In the presence of the mQGP, the real inclusive $p p$ cross section is determined by the product of the pQCD cross section and the medium modification factor $R_{p p}$ (which accounts for the jet final state interaction in the mQGP)

$$
d \sigma(p p \rightarrow h X) / d \mathbf{p}_{T} d y=R_{p p} d \sigma_{\mathrm{pert}}(p p \rightarrow h X) / d \mathbf{p}_{T} d y
$$

\footnotetext{
${ }^{1}$ We make a reasonable assumption that for $d N_{\mathrm{ch}}^{\mathrm{ue}} / d \eta$ equal to $d N_{\mathrm{ch}} / d \eta$ dynamics of the UE soft hadron production in $p p$ jet events is similar to that for $p p$ collisions without jet detecting.
} 
The first pQCD calculations of $R_{p p}$ have been performed in [29, 30] within the light-cone path integral (LCPI) approach [3] to induced gluon emission, using the measured UE charged multiplicity density for calculation of the initial mQGP temperature. The results of [30] show that for $p p$ collisions at the LHC energies medium suppression of hadron spectra can be $\sim 20-30 \%$ at $p_{T} \sim 10-20 \mathrm{GeV}$. Since $R_{p p}$ is rather close to unity and has a smooth $p_{T}$-dependence it is difficult to differentiate the scenarios with and without medium suppression using only data on the high- $p_{T}$ hadron spectra in $p p$ collisions. In [29] it was suggested to use as a signal of jet quenching in $p p$ collisions variation of the photon/hadrontagged jet fragmentation functions (FFs) with the UE multiplicity. The recent preliminary data from ALICE [31] on the jet FF modification factor $I_{p p}$ at $\sqrt{s}=5.02 \mathrm{TeV}$ for the hadron-tagget jets (with the trigger hadron momentum $8<p_{T}<15 \mathrm{GeV}$, and the associated away side hadron momentum in the range $4<p_{T}<6 \mathrm{GeV}$ ) show a monotonic decrease of $I_{p p}$ with the UE multiplicity by about $20 \%$ for the UE multiplicity density range $\sim 5-20$. This agrees qualitatively with predictions of [29]. The observation of decrease of $I_{p p}$ with the UE multiplicity, if confirmed, will be a strong argument for the scenario with mQGP formation in $p p$ jet events.

Evidently, for the scenario with mQGP formation in $p p$ collisions the theoretical $R_{A A}$, which corresponds to the experimentally measured $R_{A A}$, given by (1.1), reads

$$
R_{A A}=R_{A A}^{\mathrm{st}} / R_{p p}
$$

where $R_{A A}^{\text {st }}$ is the standard nuclear modification factor calculated with the pQCD $p p$ cross section. For a weak jet quenching in the mQGP, i.e., for $R_{p p}$ close to unity, observation of the effect of the $1 / R_{p p}$ factor in (1.3) from comparison with data on $R_{A A}$ for heavy nuclei, when $R_{A A} \sim 0.15-0.5$, is a hopeless task, because uncertainties of the present theoretical jet quenching models are rather large and the presence of $1 / R_{p p}$ in (1.3) may be mimicked by changes of the parameter values. However, the effect from $1 / R_{p p}$ factor can become more pronounced in the case of collisions of light nuclei, when $R_{A A}^{\text {st }}$ also becomes close to unity. This could potentially be verified by the future planned experimental study of jet quenching for oxygen-oxygen collisions at RHIC $(\sqrt{s}=0.2 \mathrm{TeV})[15]$ and at the LHC $[17,18](\sqrt{s}=7 \mathrm{TeV})$. In light of this, it is highly desirable to perform a quantitative analysis of jet quenching in $\mathrm{O}+\mathrm{O}$ collisions at the RHIC and LHC energies for scenarios with and without mQGP formation in $p p$ collisions.

In the present work, by fixing free parameters from fitting available data on $R_{A A}$ for heavy ion collisions, we perform calculations of the nuclear modification factor for 0.2 and $7 \mathrm{TeV} \mathrm{O}+\mathrm{O}$ collisions for scenarios with and without mQGP formation in $p p$ collisions (we will often omit for clarity adding "in $p p$ collisions"), and for an intermediate scenario when mQGP is formed only in $p p$ collisions at the LHC energies. We use the jet quenching model of [32] (with some improvements made in [11]), based on the LCPI approach [3] to induced gluon emission. We perform calculations of the radiative energy loss using the method suggested in [33], which allows to account for accurately the Coulomb effects in multiple parton scattering. An important feature of our calculations is that we use a temperature dependent parametrization of the running $\alpha_{s}$ (suggested in [34]). In [34] it was demonstrated that the $T$-dependent QCD coupling largely solves the problem of 
difference between the optimal values of $\alpha_{s}$ for the RHIC and LHC energies [11, 35]. Use of the $T$-dependent QCD coupling allows to avoid the ambiguities for small systems ( $p p$ and light ion collisions) connected with the choice of $\alpha_{s}$ for large and small size QGP, because the parameters, fitted to data for heavy ion collisions, automatically fix $\alpha_{s}$ for small size QGP. This improves the reliability of the results, as compared to our previous analyses of jet quenching in $p p$ collisions $[29,30,36]$. There we used a unique $\alpha_{s}$, which does not depend on the local QGP temperature. The disadvantage of this ansatz is that it leaves ambiguous how to choose $\alpha_{s}$ for the mQGP fireball in $p p$ collisions. Note that the issue of jet quenching in light ion collisions was addressed recently in [10] within the BDMPS formalism [2] describing multiple parton scattering in terms of the transport coefficient $\hat{q}$. However, the scenario with mQGP formation was not considered in [10].

The plan of the paper is as follows. In section 2, we briefly review the theoretical framework. In section 3, we discuss the models of the QGP fireballs in $A A$ and in $p p$ collisions used in our jet quenching calculations. In section 4 we first perform the $\chi^{2}$ fit of the experimental data from the LHC on $R_{A A}$ for $\mathrm{Pb}+\mathrm{Pb}$ and $\mathrm{Xe}+\mathrm{Xe}$ collisions for determining the optimal free parameters. Then, we present results for $R_{p p}$ and $R_{A A}$ and for the elliptic flow coefficient $v_{2}$ for heavy ion and for $\mathrm{O}+\mathrm{O}$ collisions obtained with the optimal free parameters for scenarios without and with mQGP formation. We conclude with a brief discussion of jet quenching in $p A$ collisions. Conclusions are contained in section 5 .

\section{Basic aspects of the jet quenching scheme}

Our theoretical scheme for calculation of the medium modification factors is similar to the one used in our previous jet quenching analyses (see [11, 30, 32, 34]). For this reason, here we only outline its main aspects. For $A A$ collisions we write the nuclear modification factor $R_{A A}^{\mathrm{st}}$ for given impact parameter $b$ (we consider the midrapidity region around $y=0$ )

$$
R_{A A}^{\mathrm{st}}\left(b, \mathbf{p}_{T}, y\right)=\frac{d N(A+A \rightarrow h+X) / d \mathbf{p}_{T} d y}{T_{A A}(b) d \sigma(p+p \rightarrow h+X) / d \mathbf{p}_{T} d y},
$$

where $T_{A A}(b)=\int d \boldsymbol{\rho} T_{A}(\boldsymbol{\rho}) T_{A}(\boldsymbol{\rho}-\mathbf{b})$ is the nuclear profile function, $T_{A}(\boldsymbol{\rho})=\int d z \rho_{A}\left(\sqrt{\rho^{2}+z^{2}}\right)$ is the nuclear thickness function (with $\rho_{A}$ the nuclear density). The differential yield of the process $A+A \rightarrow h+X$ in the nominator on the righthand side of (2.1) reads

$$
\frac{d N(A+A \rightarrow h+X)}{d \mathbf{p}_{T} d y}=\int d \boldsymbol{\rho} T_{A}(\boldsymbol{\rho}+\mathbf{b} / 2) T_{A}(\boldsymbol{\rho}-\mathbf{b} / 2) \frac{d \sigma_{m}(N+N \rightarrow h+X)}{d \mathbf{p}_{T} d y},
$$

where $d \sigma_{m} / d \mathbf{p}_{T} d y$ is the medium-modified cross section, given by

$$
\frac{d \sigma_{m}(N+N \rightarrow h+X)}{d \mathbf{p}_{T} d y}=\sum_{i} \int_{0}^{1} \frac{d z}{z^{2}} D_{h / i}^{m}(z, Q) \frac{d \sigma(N+N \rightarrow i+X)}{d \mathbf{p}_{T}^{i} d y} .
$$

Here $\mathbf{p}_{T}^{i}=\mathbf{p}_{T} / z$ is the transverse momentum of the initial hard parton, $d \sigma(N+N \rightarrow i+X) /$ $d \mathbf{p}_{T}^{i} d y$ is the ordinary pQCD hard cross section, and $D_{h / i}^{m}(z, Q)$ is the medium-modified FF for transition of the initial hard parton $i$, with virtuality $Q \sim p_{T}^{i}$, to the final hadron $h$. The FF $D_{h / i}^{m}$ can symbolically be written as the triple $z$-convolution

$$
D_{h / i}^{m}(Q) \approx D_{h / j}\left(Q_{0}\right) \otimes D_{j / k}^{\text {in }} \otimes D_{k / i}^{\operatorname{DGLAP}}(Q),
$$


where $D_{k / i}^{\text {DGLAP }}$ is the DGLAP parton FF for $i \rightarrow k$ transition, $D_{j / k}^{\text {in }}$ describes the inmedium $j \rightarrow k$ transition due to induced gluon emission, and $D_{h / j}$ is the ordinary vacuum FF for transition of the parton $j$ to the final hadron $h$. For $D_{h / j}\left(z, Q_{0}\right)$ we use the KKP [37] parametrization with $Q_{0}=2 \mathrm{GeV}$. We compute the DGLAP FFs $D_{k / i}^{\text {DGLAP }}$ with the help of the PYTHIA event generator [38].

The main ingredient in our analysis is the $\mathrm{FF} D_{j / k}^{\text {in }}$, which describes the medium effects. We evaluate $D_{j / k}^{\mathrm{in}}$ via the induced gluon spectrum $d P / d x$ ( $x$ is the gluon fractional momentum) in the approximation of independent gluon emission [39]. A detailed description of the method for evaluation of the induced gluon spectrum within the LCPI formalism and the implementation of the approximation of independent gluon emission for calculation of $D_{j / k}^{\text {in }}$ can be found in $[11,40]$.

The induced gluon spectrum $d P / d x$ depends on the QGP fireball density/temperature profile along the jet trajectory. Since the QGP temperature depends crucially on the size of the collision system, for a joint analysis of jet modification for large and small size collision systems it is important to account for the temperature dependence of the in-medium QCD coupling. In the present analysis we calculate the gluon induced spectrum for running temperature dependent $\alpha_{s}$. As in [34], we use the paramertization of $\alpha_{s}(Q, T)$ in the form

$$
\alpha_{s}(Q, T)= \begin{cases}\frac{4 \pi}{9 \log \left(Q^{2} / \Lambda_{\mathrm{QCD}}^{2}\right)} & \text { if } Q>Q_{f r}(T), \\ \alpha_{s}^{f r}(T) & \text { if } Q_{f r}(T) \geq Q \geq c Q_{f r}(T), \\ \alpha_{s}^{f r}(T) \times\left(Q / c Q_{f r}(T)\right) & \text { if } Q<c Q_{f r}(T),\end{cases}
$$

where $Q_{f r}(T)=\Lambda_{\mathrm{QCD}} \exp \left\{2 \pi / 9 \alpha_{s}^{f r}(T)\right\}$ (in the present analysis we take $\Lambda_{\mathrm{QCD}}=200 \mathrm{MeV}$ ). We perform calculations for $c=0.8$ and $c=0$. We take $Q_{f r}=\kappa T$, and perform fit of the free parameter $\kappa$ using data on the nuclear modification factor $R_{A A}$ for heavy ion collisions. The parametrization (2.5) with nonzero $c$ is supported by the lattice results for the in-medium $\alpha_{s}$ in the coordinate space via calculation of the free energy of a static heavy quark-antiquark pair [41]. The results of [41] give $\alpha_{s}(r, T)$ that becomes close to the ordinary vacuum QCD coupling $\alpha_{s}(Q)$ with $Q \sim 1 / r$ at $r \ll 1 / T$. In the infrared region it reaches maximum at $r \sim 1 / \kappa T$ with $\kappa \sim 4$, and then it falls to zero. With identification $r \sim 1 / Q$, this behavior is similar to that obtained in the momentum representation within the functional renormalization group [42]. The choice $c=0$ is similar to the model with $\alpha_{s}$ frozen in the infrared region $[43,44]$. Details of incorporating the $T$-dependence of $\alpha_{s}$ into the LCPI scheme can be found in [34]. It should be emphasized that the use of the running $\alpha_{s}$ is very important in dealing with jet quenching simultaneously in a large size QGP for heavy ion collisions and in a smaller QGP for light ion and $p p$ collisions, because the typical virtuality in induced gluon emission increases with decreasing medium size. The growth of the virtuality scale from heavy ion to $p p$ collisions turns out to be quite large (by a factor of $\sim 2[30])$.

We calculate the induced gluon spectrum with the Debye mass obtained in the lattice analysis [45], and take $m_{q}=300$ and $m_{g}=400 \mathrm{MeV}$ for the light quark and gluon quasiparticle masses in the QGP [46]. In numerical calculations for the T-dependence of the 
Debye mass (and of $\alpha_{s}(Q, T)$ ) we define the temperature via the lattice entropy density $s(\tau)$ obtained in [47]. For a given fireball entropy, this procedure gives $T$ which is somewhat larger than the ideal gas temperature.

For evaluation of $R_{A A}$ for a given centrality range $\Delta c$, we perform accurate averaging over the geometry of $A A$ collision and over the jet trajectories in the medium. As in [32] we performed calculations for a QGP fireball with a uniform density distribution in the transverse plane, and with the Bjorken 1+1D longitudinal expansion [48]. The approximation of a uniform fireball is a crucial simplification, because in this case the medium density profile along the fast parton trajectory is independent of the jet production point (only its length $L$ varies). This approximation greatly reduces the computational time, since we can first tabulate the $L$-dependence of the induced gluon spectrum, and then to use it in computations of the FFs for arbitrary geometry of the jet production.

We evaluate the medium modification factor $R_{p p}$ for $p p$ collisions in a similar way. However, for $p p$ collisions we need only the medium modification factor for the whole range of the impact parameter. The azimuthal dependence of jet quenching in mQGP is irrelevant for calculation of $R_{p p}$. Therefore, averaging over the geometry of the $p p$ collision is simply reduced to averaging of the medium modified FFs over the parton path length $L$ in the medium. We perform averaging over $L$ for a symmetric fireball of radius $R_{f}$ produced in the central $p p$ collision. This procedure seems to be reasonable, since the jet production should be dominated by the head-on collisions, and the azimuthal asymmetry is anyway irrelevant for $R_{p p}$. We evaluate the $L$-distribution using the MIT bag model quark density (we assume that quarks and gluons have similar distributions in the transverse coordinates). The $L$-distribution turns out to be strongly peaked around $L \sim R_{f}$. For this reason the $L$-fluctuations lead to a small change of $R_{p p}$ as compared to the $R_{p p}$ calculated for $L=R_{f}$.

As in our previous jet quenching analyses, we calculate the parton hard cross sections in the LO pQCD with the CTEQ6 [49] parton distribution functions. For nuclei we use the nuclear parton distributions with the EPS09 correction [50]. To simulate the higher order effects we take for the virtuality scale in $\alpha_{s}$ the value $a Q$ with $a=0.265$ (as in the PYTHIA event generator [38]). This gives for $p p$ collisions a fairly good description of the $p_{T}$-dependence of the hadron spectra for the scenario without mQGP formation. In principle, for scenario with mQGP formation in $p p$ collisions the perturbative cross section in formula (1.2) should differ from that for the scenario without jet quenching due to presence of $R_{p p}$ on the righthand side of (1.3). For a fully consistent treatment of $R_{p p}$ one should use a bootstrap procedure with adjusting the free parameters to fit the experimental $p p$ cross section. However, this consistent procedure does not make much sense (at least at this stage). The point is that the hadron spectra have a very steep $p_{T}$-dependence (as compared to a weak $p_{T}$-dependence of $R_{p p}$ ). For this reason $R_{p p}$ (and $R_{A A}$ ) is only weakly sensitive to possible difference in the perturbative cross sections for scenarios without and with jet quenching in $p p$ collisions. To roughly mimic the change of the pQCD $p p$ cross section in scenario with mQGP, we performed calculations of $R_{p p}$ and $R_{A A}$ using for the $\alpha_{s}$ virtuality scale in the hard cross sections $a^{\prime} Q$ with $a^{\prime}=0.13$ for the LHC energies and $a^{\prime}=0.205$ for the RHIC energy $\sqrt{s}=0.2 \mathrm{TeV}$. These values of $a^{\prime}$ give the $p_{T^{-}}$-dependence of 
the righthand side of (1.2) which is reasonably close to that for perturbative cross section without jet quenching for the hard scale $a Q$ with the PYTHIA prescription $a=0.265$ (which we use for scenario without mQGP formation). However, we have found that the effect of the modification of the hard cross section for the mQGP version on our predictions for $R_{p p}$ and $R_{A A}$ is very small, as expected.

As in [32], we treat the effect of the collisional energy loss, which is relatively small [51-53], as a small perturbation to the radiative jet quenching. We incorporate it in calculating the FFs $D_{j / k}^{\text {in }}$ by renormalizing the initial QGP temperature according to the relation

$$
\Delta E_{\mathrm{rad}}\left(T_{0}^{\prime}\right)=\Delta E_{\mathrm{rad}}\left(T_{0}\right)+\Delta E_{\mathrm{col}}\left(T_{0}\right)
$$

where $\Delta E_{\mathrm{rad} / \mathrm{col}}$ is the radiative/collisional energy loss, $T_{0}$ is the true initial QGP temperature, and $T_{0}^{\prime}$ is the new temperature.

We conclude this section with a discussion of the possible errors due to the neglect of the transverse QGP motion and the use of a flat initial entropy density. We begin with the errors due to the neglect of the QGP transverse flow. It was shown long ago by Baier, Mueller and Schiff [54], within the BDMPS formalism [2], that the flow corrections to predictions obtained using the Bjorken model should be small. This occurs due to an almost complete compensation between the enhancement of parton energy loss caused by increase of the fireball size and its suppression caused by reduction of the transport coefficient due to the QGP expansion. More recently, a detailed numerical analysis of the flow effects in jet quenching at the RHIC and LHC energies, based on the pQCD ansatz for the radiative parton energy loss, performed by Betz and Gyulassy [55] confirmed that the effect of the QGP transverse expansion on the medium suppression of high- $p_{T}$ spectra is small. In [55] it was found that for $0.2 \mathrm{TeV} \mathrm{Au}+\mathrm{Au}$ and $2.76 \mathrm{TeV} \mathrm{Pb}+\mathrm{Pb}$ collisions the change of $R_{A A}$ due to the transverse flow is $\lesssim 1-2 \%$ (see figure 2 of [55]), and it holds both for central and noncentral collisions. The results of [55] show that for heavy ion collisions the errors of our predictions due to the neglect of the QGP transverse expansion should be small. It is important that for noncentral $0.2 \mathrm{TeV} \mathrm{Au}+\mathrm{Au}$ collisions for $20-30 \%$ centrality bin the authors of [55] obtained very small flow corrections to $R_{A A}$ both for the jet azimuthal angle $\phi \sim 0$ and $\phi \sim \pi / 2$ (i.e., for the jet directions along the short and long fireball semi-diameters). For $\mathrm{Au}+\mathrm{Au}$ collisions in $20-30 \%$ centrality bin the short fireball semi-diameter is $\sim 2.5 \mathrm{fm}$. Since this size is close to the oxygen radius, one can conclude that the flow correction to $R_{A A}$ in $\mathrm{O}+\mathrm{O}$ collisions should be small. For the mQGP produced in $p p$ collisions the fireball radius (see eq. (3.4) below) is smaller than the short fireball semi-diameter for $\mathrm{Au}+\mathrm{Au}$ collisions for $20-30 \%$ centrality by just $\sim 40 \%$. Due to the smaller QGP temperature (see eq. (3.5) below) the QGP sound velocity for $p p$ collisions should be smaller than that for $\mathrm{Au}+\mathrm{Au}$ collisions. This can reduce somewhat the transverse QGP velocity (which is approximately proportional to the QGP squared sound velocity [56]) for $p p$ collisions. The above facts show that the magnitudes of the QGP transverse flow for $0.2 \mathrm{TeV} \mathrm{Au}+\mathrm{Au}$ collisions for $20-30 \%$ centrality bin and $p p$ collisions should not differ drastically. Therefore, there is no reason to expect that the flow correction to $R_{p p}$ can be dramatically larger than that found in [55] for $R_{A A}$ in noncentral $\mathrm{Au}+\mathrm{Au}$ 
collisions. Thus, one can conclude that the errors of our predictions due to the use of the Bjorken model should be quite small.

The situation with the errors due to the use a flat initial entropy distribution in some sense is similar to that for the neglect of the transverse flow. In this case one can also expect a strong compensation between the enhancement of the parton energy loss in the regions where the density is overestimated and its suppression in the regions where the density is underestimated. The jet configurations that can potentially lead to the incomplete compensation are the jet production near the surface of the fireball, when $R_{A A} \sim 1$ for the jets heading outward, and $R_{A A} \ll 1$ for the ones heading to the core of the fireball. This nonlinear effect is possible for heavy ion collisions, but cannot be important for small systems. In general, it is clear that for small systems the sensitivity of jet quenching to the form of the fireball density should be weak, since for such systems we have a situation where the typical formation length for induced gluon emission is of the order of the fireball size or larger. In such a regime the form of the density profile along the fast parton trajectory is not very important, and the parton energy loss is mostly controlled by the total amount of the matter traversed by fast parton. As far as the possible errors in $R_{A A}$ for heavy ion collisions are concerned, even if they are several times larger than the flow corrections found in [55], they can lead only to a relatively small change of the parameters fitted from heavy ion data. For instance, for $R_{A A} \sim 0.2$ a change of $R_{A A}$ by $\sim 20 \%$ can be mimicked by changing $\alpha_{s}$ by $\sim 3 \% .^{2}$ Note that the necessary change of $\alpha_{s}$ to compensate the errors $\sim 20 \%$ in $R_{A A}$ for heavy ion collisions will translate to the errors $\lesssim 10 \%$ in $R_{A A, p p}-1$ for small system. Thus, it is clear that the approximation of a flat QGP density cannot spoil significantly the quality of the extrapolation of the jet quenching predictions from heavy ion collisions to collisions of small systems. It is worth noting that, in principle, even for an accurate hydrodynamic fireball evolution it is impossible to avoid the errors of the order of $\sim 10 \%$ in $R_{A A, p p}-1$ in extrapolating the theoretical predictions from heavy ion collisions to small system collisions, because there are also other considerable theoretical uncertainties in jet quenching calculations (e.g. related to the treatment of multiple gluon emission and to the choice of the number density of the color centers, which is the basic input to calculation of the induced gluon spectrum).

\section{Models of the QGP fireballs for $A A$ and $p p$ collisions}

We perform calculations for simple Bjorken's 1+1D expansion [48] of the QGP. It gives the entropy density $s(\tau) / s\left(\tau_{0}\right)=\tau_{0} / \tau$, where $\tau_{0}$ is the thermalization time. At $\tau<\tau_{0}$ we use a linear parametrization $s(\tau)=s\left(\tau_{0}\right) \tau / \tau_{0}$. We set $\tau_{0}=0.5 \mathrm{fm}$ both for $A A$ and $p p$ collisions. But to understand sensitivity of the results to $\tau_{0}$, we also perform calculations for $\tau_{0}=0.8 \mathrm{fm}$. As in our previous jet quenching analyses, we model QGP by a system of the static Debye screened color centers [1]. We use the ideal gas model to relate the number density of the QGP constituents to the entropy density. This gives the effective number

\footnotetext{
${ }^{2}$ This estimate can be easily obtained from the analytical relation between the energy loss and the medium suppression factor derived in [39], considering that the radiative energy loss is dominated by the $N=1$ rescattering term (which is $\propto \alpha_{s}^{3}$ ).
} 
density of the triplet color centers $n(z)=b T^{3}(z)$ with $b=9 \xi(3)\left(N_{f}+4\right) / \pi^{2} \approx 7.125$ (for $\left.N_{f}=2.5\right)$. As was said above, we use the fireball model with a uniform density distribution in the transverse plane.

\subsection{The QGP fireball in $A A$ collisions}

Let us start with $A A$ collisions. To fix the average initial QGP entropy density for $A A$ collisions we use the relation [48]

$$
s_{0}=\frac{C}{\tau_{0} S_{f}} \frac{d N_{\mathrm{ch}}(A A)}{d \eta},
$$

where $S_{f}$ is the area of the overlap region of two colliding nuclei, and $C=d S / d y /$ $d N_{\mathrm{ch}}(A A) / d \eta \approx 7.67[57]$ is the entropy/multiplicity ratio. We calculate $d N_{\mathrm{ch}}(A A) / d \eta$ in the Glauber wounded nucleon model [58] with parameters of the model as in our Monte-Carlo Glauber analyses [59, 60], which describe very well data on the midrapidity $d N_{\mathrm{ch}} / d \eta$ in $0.2 \mathrm{TeV} \mathrm{Au}+\mathrm{Au}$ [61], 2.76 [62] and 5.02 TeV [63] $\mathrm{Pb}+\mathrm{Pb}$, and $5.44 \mathrm{TeV}$ $\mathrm{Xe}+\mathrm{Xe}[64]$ collisions. For heavy nuclei we use the Woods-Saxon nuclear density $\rho_{A}(r)=$ $\rho_{0} /\left[1+\exp \left(\left(r-R_{A}\right) / d\right)\right]$. For $\mathrm{Pb}$ nucleus we take the parameters $R_{A}=6.62$ and $d=0.546 \mathrm{fm}$, as in the PHOBOS Glauber model [65], and for Xe nucleus we take $d=0.54 \mathrm{fm}$ and $R_{A}=\left(1.12 A^{1 / 3}-0.86 / A^{1 / 3}\right) \mathrm{fm}$, as in the GLISSANDO Glauber model [66] (it gives $R_{A} \approx 5.49 \mathrm{fm}$ ). For our basic version we define $S_{f}$ for overlap of two circles with radius $R=R_{A}+k d$ with $k=2\left(R_{A}\right.$ and $d$ are the parameters of the Woods-Saxon nuclear density). To understand the sensitivity of the results to the choice of $k$, we also performed calculations for $k=3$. The range $2 \lesssim k \lesssim 3$ seems to be reasonable for determining the fireball size, because, on the one hand, it guarantees that the fraction of the lost QGP volume is small. On the other hand, for such values of $k$ our ansatz with a flat fireball density does not lead to a considerable redistribution of the QGP density into regions where the true QGP density should vanish. Our calculations give a small variation of $R_{A A}$ in heavy ion collisions with $k$ in the range $2 \lesssim k \lesssim 3$ (see below).

For $\mathrm{O}+\mathrm{O}$ collisions we performed calculations for the harmonic oscillator shell model nuclear density

$$
\begin{aligned}
\rho_{A}(r) & =\frac{4}{\pi^{3 / 2} r_{A}^{3}}\left[1+\frac{A-4}{6}\left(\frac{r}{r_{A}}\right)^{2}\right] \exp \left(r^{2} / r_{A}^{2}\right), \\
r_{A}^{2} & =\left(\frac{5}{2}-\frac{4}{A}\right)^{-1}\left(\left\langle r_{\mathrm{ch}}^{2}\right\rangle_{A}-\left\langle r_{\mathrm{ch}}^{2}\right\rangle_{p}\right)
\end{aligned}
$$

with $\left\langle r_{\mathrm{ch}}^{2}\right\rangle_{A}=7.29 \mathrm{fm}^{2}$ for ${ }^{16} \mathrm{O}$ and $\left\langle r_{\mathrm{ch}}^{2}\right\rangle_{p}=0.7714 \mathrm{fm}^{2}$ [66]. Also, to understand the sensitivity of the results to the ${ }^{16} \mathrm{O}$ nuclear density we have performed calculations for the Woods-Saxon density. For the Woods-Saxon oxygen nuclear density we take $d=0.513 \mathrm{fm}$ [67] and $R_{A}=2.2 \mathrm{fm}$ (fixed through the condition of equal $\left\langle r_{\mathrm{ch}}^{2}\right\rangle$ for the Woods-Saxon and the harmonic oscillator shell model nuclear density (3.2)). Our calculations show that the difference between $R_{A A}$ for the harmonic oscillator shell model and for the Woods-Saxon one is very small for the whole range of centrality. We therefore present 
results only for the oscillator shell model nuclear density. Similarly to heavy ion collisions, for $\mathrm{O}+\mathrm{O}$ collisions we define $S_{f}$ for overlap of two circles with radius $R=R_{A}+k d$ and $k=2$, where $R_{A}$ and $d$ the parameters of the Woods-Saxon nuclear density (both for the harmonic oscillator shell model density and the Woods-Saxon one). For heavy ions the results are not very sensitive to variation of the nuclear geometry parameter $k$. But for light nuclei the variation of $R_{A A}$ with $k$ may be more noticeable, since the ratio of the nuclear surface thickness to the nuclear radius becomes larger. To illustrate dependence of the result on $k$, for $\mathrm{O}+\mathrm{O}$ collisions we also present results for $k=3$.

Figure 1 shows the centrality dependence of the initial QGP temperature obtained within the Glauber model with the help of the relation (3.1) for heavy ion and $\mathrm{O}+\mathrm{O}$ collisions. We show the results for the ideal gas model, when $s(T)=a T^{3}$ with $a=$ $\frac{4 \pi^{2}}{15}\left(8 / 3+7 N_{f} / 4\right)\left(a \approx 18.53\right.$, if one takes $\left.N_{f}=2.5\right)$. The QGP temperature calculated via (3.1) for the lattice entropy density [47] is somewhat bigger than that for the ideal gas model (by $\sim 5-10 \%$ at $T \sim 300-450$, and by $\sim 15-25 \%$ at $T \sim 150-200 \mathrm{MeV}$ ). From figure 1 one can see that for $7 \mathrm{TeV} \mathrm{O}+\mathrm{O}$ collisions the initial fireball temperature is qualitatively similar to that for $0.2 \mathrm{TeV} \mathrm{Au+Au}$ collisions. We therefore expect the QGP formation and jet quenching in $7 \mathrm{TeV} \mathrm{O}+\mathrm{O}$ collisions. However, for $0.2 \mathrm{TeV} \mathrm{O}+\mathrm{O}$ collisions the initial temperature for centrality $\lesssim 30 \%$ is comparable to that expected for $p p$ collisions (see below). For this reason, there is some doubt whether QGP formation in $0.2 \mathrm{TeV} \mathrm{O}+\mathrm{O}$ collisions is possible, if there is no mQGP formation in $p p$ collisions. But one should bear in mind that $\mathrm{O}+\mathrm{O}$ collisions have better conditions for collectivity in the final state, because in this case the Knudsen number becomes bigger by a factor of 2 as compared to that for $p p$ collisions. In the present analysis we assume that in $0.2 \mathrm{TeV} \mathrm{O}+\mathrm{O}$ collisions a QGP is formed.

For a flat entropy distribution the model with the almond shaped fireball with two cups somewhat overestimates the anisotropy coefficient $\epsilon_{2}$, as compared to that calculated with an accurate $b$-dependent entropy density [11]. This fact is immaterial for $R_{A A}$, which is practically insensitive to azimuthal asymmetry of the fireball. But for calculation of the flow coefficient $v_{2}$ this model is too crude. In order to have in our calculations the fireball ellipticity which coincides with that for the accurate Glauber model fireball density, as in [11], we transform the almond shaped region into an elliptic one (of the same area). We perform calculations of $v_{2}$ for two choices of the fireball eccentricity $\epsilon_{2}$. For the first variant, we calculate $\epsilon_{2}$ in the optical Glauber wounded nucleon model, and for the second variant we use $\epsilon_{2}$ obtained in the Monte-Carlo Glauber model of [59,60]. The Monte-Carlo version gives $\epsilon_{2}$, which, contrary to the optical model one, does not vanish for central collisions (due to density fluctuations). But for noncentral collisions of heavy nuclei predictions of the optical and Monte-Carlo Glauber models for $\epsilon_{2}$ do not differ significantly. Our treatment of the flow coefficient $v_{2}$ for the Monte-Carlo version of $\epsilon_{2}$ ignores possible decorrelation between the participant plane and the true reaction plane. The effect of this decorrelation should be small for heavy nuclei (see discussion in [11]). However, for light ion collisions, such as $\mathrm{O}+\mathrm{O}$ in our case, when the density fluctuations become large, our calculations of $v_{2}$ become clearly less robust. It worth noting that for light nuclei even the initial anisotropy coefficient $\epsilon_{2}$ itself cannot be calculated accurately in the Monte-Carlo Glauber model, 


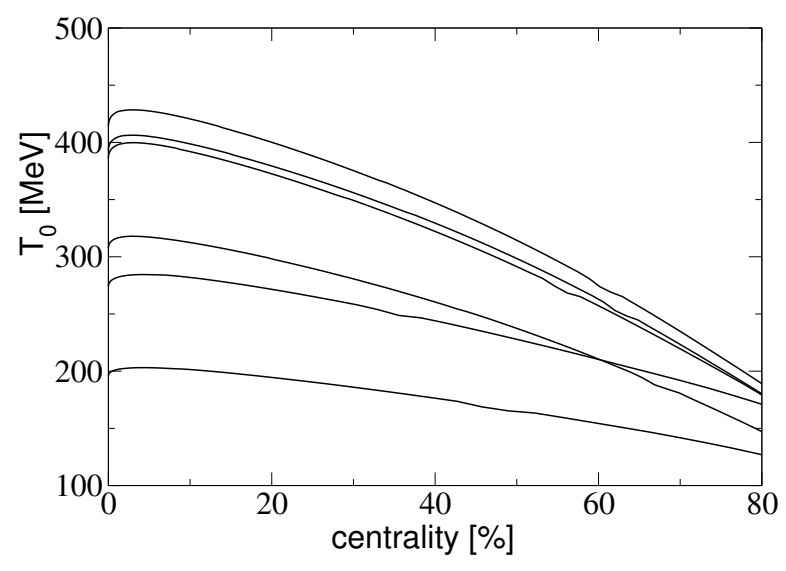

Figure 1. Centrality dependence of the initial fireball temperature at $\tau_{0}=0.5 \mathrm{fm}$ for the ideal gas model obtained in the Glauber model via the average entropy density for (from top to bottom at low centrality): 5.02 and $2.76 \mathrm{TeV} \mathrm{Pb}+\mathrm{Pb}, 5.44 \mathrm{TeV} \mathrm{Xe}+\mathrm{Xe}, 0.2 \mathrm{TeV} \mathrm{Au}+\mathrm{Au}, 7$ and $0.2 \mathrm{TeV}$ $\mathrm{O}+\mathrm{O}$ collisions.

because the theoretical predictions become sensitive to the size of the entropy sources in the whole range of centrality. For this reason, our results for $v_{2}$ in $\mathrm{O}+\mathrm{O}$ collisions are of illustrative character. But nevertheless they allow to understand the relation between the initial fireball ellipticity $\epsilon_{2}$ and the flow coefficient $v_{2}$ for light nucleus collisions. We perform calculation of $v_{2}$ using $\epsilon_{2}$ for the Gaussian sources with width $0.7 \mathrm{fm}$. In figure 2 we show the theoretical prediction for $\epsilon_{2}$ for 0.2 and $7 \mathrm{TeV} \mathrm{O}+\mathrm{O}$ collisions, obtained in the optical Glauber wounded nucleon model and in the Monte-Carlo one, which we use in our calculations of $v_{2}$. To illustrate the sensitivity of $\epsilon_{2}$ to the source width in the Monte-Carlo scheme we also plot in figure 2 predictions for the Gaussian width $0.4 \mathrm{fm}$. From figure 2 one sees that there is a significant difference between the results for the optical and MonteCarlo versions, and that the Monte-Carlo model $\epsilon_{2}$ is rather sensitive to the entropy source size. The curves for $\epsilon_{2}$ for heavy ion collisions can be found in [11].

For $A A$ collisions we use the medium life/freeze-out time $\tau_{f . o .} \approx 1.05 \times\left(d N_{\mathrm{ch}} / d \eta\right)^{1 / 3}$, which is supported by the pion interferometry at RHIC [68] and LHC [69]. However, the exact value of the QGP fireball life time is not very crucial to our analysis, since the jet quenching effect of the final stage with $\tau \sim \tau_{\text {f.o. }}$ is very small [11, 32]. Note that, from the viewpoint of jet quenching, the nature of the medium in the final stage, whether it is QGP or hadron gas, is not vary crucial, because for a given entropy the energy loss in QGP and hadronic gas turn out to be close to each other [70].

\subsection{The mQGP fireball parameters for $p p$ collisions}

We now come to the mQGP fireball in $p p$ collisions. Our treatment of the mQGP fireball is similar to that of ref. [30]. To fix $T_{0}$, we use the relation (3.1) with $d N_{\mathrm{ch}}(A A) / d \eta$ replaced by the UE multiplicity density in $p p$ collisions $d N_{\mathrm{ch}}^{\text {ue }}(p p) / d \eta$. We take $S_{f}=\pi R_{f}^{2}$, where $R_{f}$ is the effective radius of the mQGP fireball in the $p p$ collision (effective in the sense that it is an average radius for the whole range of the impact parameter). We determine $R_{f}$ via the UE multiplicity density $d N_{\mathrm{ch}} / d \eta$ using the prediction for $R_{f}$ obtained in numerical 


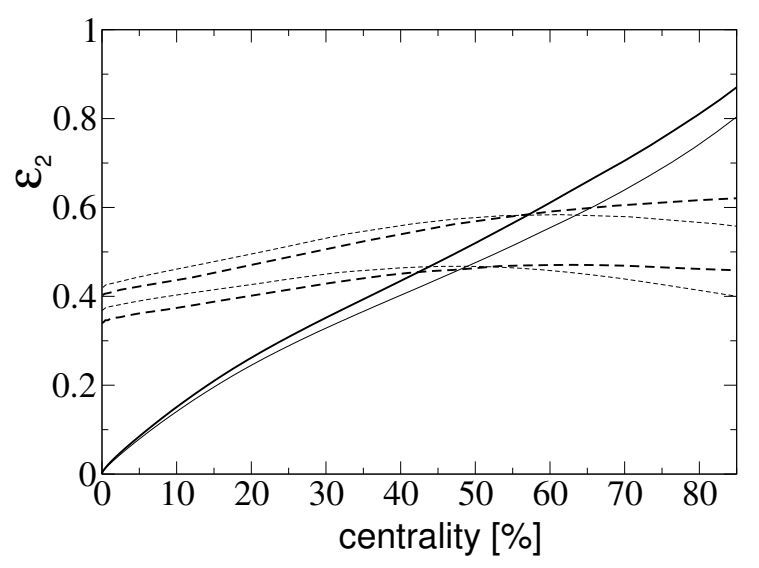

Figure 2. Centrality dependence of the initial fireball eccentricity $\epsilon_{2}$ for $\mathrm{O}+\mathrm{O}$ collisions at $\sqrt{s}=0.2 \mathrm{TeV}$ (thin lines) and $7 \mathrm{TeV}$ (thick lines) calculated in the optical (solid) and in the MonteCarlo (dashed) Glauber wounded nucleon model. The curves for the Monte-Carlo version are obtained for the Gaussian width parameter of the entropy sources (top to bottom) 0.4 and $0.7 \mathrm{fm}$.

simulations performed in [71] within the IP-Glasma model [72]. We use the parametrization of $R_{f}$ from [71] as a function of $d N_{g} / d y$ given in [73]

$$
\begin{aligned}
R_{f} & =1 \mathrm{fm} \times f_{p p}\left(\sqrt[3]{d N_{g} / d y}\right), \\
f_{p p}(x) & = \begin{cases}0.387+0.0335 x+0.274 x^{2}-0.0542 x^{3} & \text { if } x<3.4 \\
1.538 & \text { if } x \geq 3.4\end{cases}
\end{aligned}
$$

We take $d N_{g} / d y=b d N_{\mathrm{ch}} / d \eta$ with $b=C 45 / 2 \pi^{4} \xi(3) \approx 2.13$. For $p p$ collisions at $\sqrt{s}=0.2$ we calculate the UE multiplicity density using the $K_{\text {ue }}$ from PHENIX measurement [21] and the minimum bias non-single-diffractive event multiplicity density $d N_{\mathrm{ch}}^{\mathrm{mb}} / d \eta=2.65$ from the UA1 Collaboration [74]. In the plateau region this gives $d N_{\mathrm{ch}}^{\mathrm{ue}} / d \eta \approx 5.79$. We evaluate the UE multiplicity at $\sqrt{s}=2.76$ and $5.02 \mathrm{TeV}$ by interpolating the ATLAS data [22] between $\sqrt{s}=0.9$ and $7 \mathrm{TeV}$ that give in the plateau region $d N_{\mathrm{ch}}^{\text {ue }} / d \eta \approx 7.5$ and 13.9 . Assuming that $d N_{\mathrm{ch}}^{\text {ue }} / d \eta \propto s^{\delta}$, we obtained $d N_{\mathrm{ch}}^{\text {ue }} / d \eta \approx 10.5(12.6)$ for $\sqrt{s}=2.76(5.02) \mathrm{TeV}$. Using these values of $d N_{\mathrm{ch}}^{\mathrm{ue}} / d \eta$ we obtain

$$
R_{f}[\sqrt{s}=0.2,2.76,5.02,7 \mathrm{TeV}] \approx[1.26,1.44,1.49,1.51] \mathrm{fm} .
$$

Then, using the above values of $d N_{\mathrm{ch}}^{\mathrm{ue}} / d \eta$ and $R_{f}$ from (3.4), we obtain for the initial temperature of the mQGP fireball for the ideal gas entropy and for the lattice entropy [47] (numbers in brackets)

$$
T_{0}[\sqrt{s}=0.2,2.76,5.02,7 \mathrm{TeV}] \approx[195(226), 217(247), 226(256), 232(261)] \mathrm{MeV} .
$$

As one can see, for the lattice entropy $T_{0}$ is larger by $\sim 10-15 \%$ than $T_{0}$ determined in the ideal gas model. 


\section{Numerical results}

\subsection{Optimal $\kappa$ from the $\chi^{2}$ fit}

We have adjusted the free parameter $\kappa$ by $\chi^{2}$ fitting of the LHC data on $R_{A A}$ for heavy ions collisions for centrality $\lesssim 30 \%$ and the transverse momentum $10<p_{T}<120 \mathrm{GeV}$. We have used data from ALICE [75], ATLAS [76], and CMS [77] for $2.76 \mathrm{TeV} \mathrm{Pb}+\mathrm{Pb}$ collisions; from ALICE [78], ATLAS [79], and CMS [80] for $5.02 \mathrm{TeV} \mathrm{Pb+Pb} \mathrm{collisions;}$ and for 5.44 TeV Xe+Xe collisions from ALICE [81], ATLAS [82], and CMS [83]. As usual, we define $\chi^{2}$ as

$$
\chi^{2}=\sum_{i}^{N} \frac{\left(f_{i}^{\exp }-f_{i}^{\mathrm{th}}\right)^{2}}{\sigma_{i}^{2}},
$$

where $N$ is the number of the data points, and $\sigma_{i}^{2}=\sigma_{i \text {,stat }}^{2}+\sigma_{i \text {,sys }}^{2}$. We calculate $\chi^{2}$ using the theoretical $R_{A A}$ obtained with the help of a cubic spline interpolation in $\kappa$ using a pre-prepared grid with steps $\Delta \kappa / \kappa \sim 0.1$. The optimal values of $\kappa$, and the corresponding $\chi^{2} /$ d.p. ( $\chi^{2}$ per data point), for the versions with and without mQGP formation in $p p$ collisions, obtained using the geometrical parameter $k=2$ and the parametrization (2.5) with $c=0.8$, are summarized in table 1 . In table 1 we present results for each energy (and process) separately and for the combined fit for all LHC data. We show for $\kappa$ the standard errors (i.e. corresponding to $\Delta \chi^{2}=1$ ), and the $95 \%$ confidence intervals (CIs) for the $95 \%$ quantile of the $\chi^{2}$-distribution. From table 1 it is seen that for all the cases we have a quite good fit quality $\left(\chi^{2} /\right.$ d.p. $\left.\lesssim 1\right)$. The optimal values of $\kappa$ for different energies/processes are very similar. We find, as one could expect, that scenario with mQGP formation requires somewhat smaller values of $\kappa$ (the reduction of $\kappa$ for this version is $\sim 1$ ). We have also performed fitting for the geometrical parameter $k=3$. In this case, for the optimal $\kappa$ for all the LHC data we obtained $\kappa \approx 3.74(2.98)$ for scenarios without(with) mQGP formation with a quite good fit quality $\left(\chi^{2} /\right.$ d.p. $\left.\sim 0.7\right)$. Our fit for $\tau_{0}=0.8 \mathrm{fm}$ gives the values of $\kappa$ that are smaller by $\sim 0.22(0.12)$ for scenarios without(with) mQGP formation. For calculations with $c=0$ in (2.5), the optimal values of $\kappa$ are larger than that for $c=0.8$ by $\sim 0.4(0.05)$ for versions without(with) mQGP formation.

\subsection{Predictions for $R_{p p}, R_{A A}$ and azimuthal asymmetry $v_{2}$}

The results for $R_{p p}$, obtained for the optimal value $\kappa=2.55$ for $c=0.8$, are shown in figure 3 for the RHIC energy $\sqrt{s}=0.2$, and the LHC energies $\sqrt{s}=2.76,5.02$, and $7 \mathrm{TeV}$. To illustrate sensitivity of the results to the value of $\tau_{0}$, we show in figure 3 the results for $\tau_{0}=0.8 \mathrm{fm}$. As one can see, the difference between the results for $\tau_{0}=0.5$ and $0.8 \mathrm{fm}$ is rather small, especially for the LHC energies. The curves for $\tau_{0}=0.8 \mathrm{fm}$ in figure 3 are obtained with the optimal $\kappa$ for $R_{A A}$ for $\tau_{0}=0.5 \mathrm{fm}$. The results for $R_{p p}$ obtained for the value of $\kappa$ adjusted from fit of $R_{A A}$ with $\tau_{0}=0.8 \mathrm{fm}$ are very close to that shown in figure 3 for $\tau_{0}=0.5 \mathrm{fm}$. The curves shown in figure 3 correspond to our fit of the heavy ion data with the geometrical parameter $k=2$. Calculations of $R_{p p}$ with $\kappa \approx 2.98$ obtained from the heavy ion fit with $k=3$ give a small ( $\lesssim 5 \%$ ) decrease of $1-R_{p p}$. Thus, our results for $R_{p p}$ are quite stable against variation of the parameter $k$ used for calculations of $R_{A A}$ in heavy 


\begin{tabular}{|c|ccc|ccc|}
\hline & \multicolumn{3}{|c|}{ Without mQGP } & \multicolumn{3}{c|}{ With mQGP } \\
\cline { 2 - 7 } & $\kappa$ & $95 \%$ CI & $\chi^{2} / d . p$. & $\kappa$ & $95 \%$ CI & $\chi^{2} / d . p$. \\
\hline $\mathrm{Pb}+\mathrm{Pb} 2.76 \mathrm{TeV}$ & $3.44_{-0.04}^{+0.04}$ & $(3.16,3.7)$ & 0.75 & $2.57_{-0.05}^{+0.05}$ & $(2.3,2.82)$ & 1 \\
\hline $\mathrm{Pb}+\mathrm{Pb} 5.02 \mathrm{TeV}$ & $3.47_{-0.06}^{+0.06}$ & $(3.02,3.82)$ & 0.76 & $2.5_{-0.08}^{+0.08}$ & $(2.1,2.8)$ & 1 \\
\hline $\mathrm{Xe}+\mathrm{Xe} 5.44 \mathrm{TeV}$ & $3.59_{-0.08}^{+0.08}$ & $(2.92,4.19)$ & 0.47 & $2.52_{-0.12}^{+0.109}$ & $(2,3.3)$ & 0.51 \\
\hline All LHC data & $3.47_{-0.03}^{+0.03}$ & $(3.13,0.3 .78)$ & 0.68 & $2.55_{-0.04}^{+0.04}$ & $(2.16,2.88)$ & 0.87 \\
\hline
\end{tabular}

Table 1. Optimal values of $\kappa$ with $1 \sigma$ standard errors, $95 \%$ CI and corresponding $\chi^{2} / d . p$., obtained for $k=2, c=0.8$ at $\tau_{0}=0.5 \mathrm{fm}, \tau_{\max }=1.05 \times\left(d N_{\mathrm{ch}} / d \eta\right)^{1 / 3}$ for $10<p_{T}<120 \mathrm{GeV}$ for different energies/processes separately and for all LHC data for scenarios without (left) and with (right) mQGP formation in $p p$ collisions.

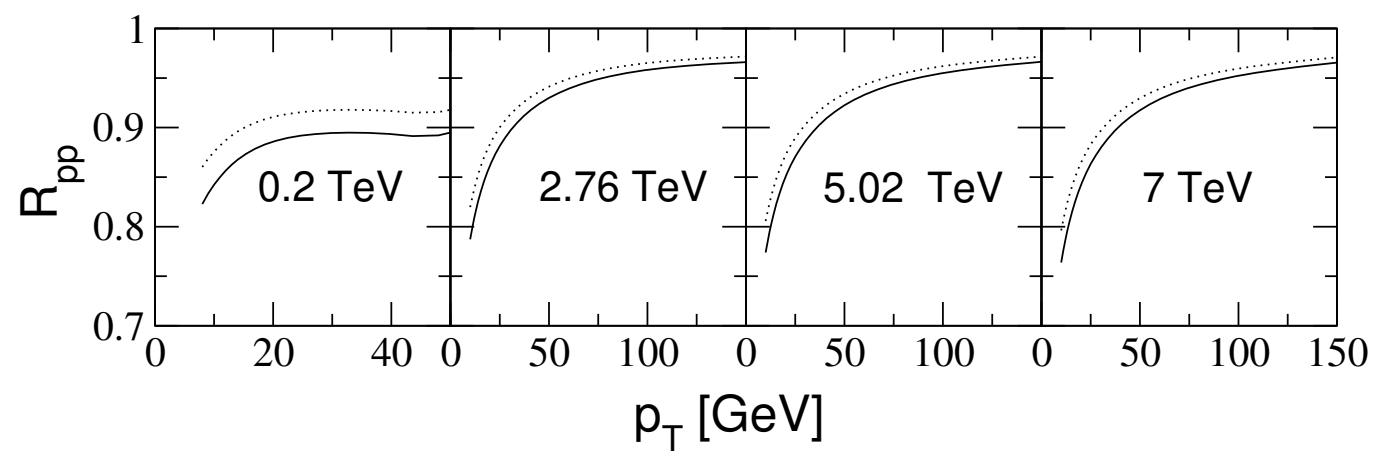

Figure 3. $R_{p p}$ of charged hadrons for $0.2,2.76,5.02$ and $7 \mathrm{TeV} p p$ collisions from our calculations for $\tau_{0}=0.5$ (solid) and $0.8 \mathrm{fm}$ (dotted) with $\kappa=2.55$ obtained by fitting all the LHC data on $R_{A A}$ for heavy ion collisions with the geometrical parameter $k=2$.

ion collisions. Note that the specific form (3.3) of the fireball radius $R_{f}$ from the IP-Glasma model, used in our calculations, is practically unimportant for $R_{p p}$, because, at a given UE multiplicity density, it varies very slowly with $R_{f}$. We observed that at $p_{T} \sim 10-20 \mathrm{GeV}$ the variation of $R_{p p}$ with the fireball radius for the interval $R_{f} / 1.3-R_{f} 1.3$ (with $R_{f}$ given by (3.4)) is $\lesssim 2 \%$, and at $p_{T} \gtrsim 50 \mathrm{GeV}$ it becomes $\lesssim 0.3 \%$. Note that the $R_{p p}$ obtained in the present work is closer to 1 than the band for $R_{p p}$ given in our previous analysis [30]. There calculations were performed for a temperature independent $\alpha_{s}^{f r}$, invariable in the whole QGP fireball. This entails some arbitrariness in the predictions for $R_{p p}$ as different choices of $\alpha_{s}^{f r}$ for large and small size QGPs are possible.

In figures 4-6 we compare our results for $R_{A A}$ for charged hadrons with the LHC data for 2.76 and $5.02 \mathrm{TeV} \mathrm{Pb}+\mathrm{Pb}$ and $5.44 \mathrm{TeV} \mathrm{Xe}+\mathrm{Xe}$ collisions used in our $\chi^{2}$ fitting. We show the curves for the optimal values of $\kappa$ for the scenarios with and without mQGP formation. In figures $4-6$ we show the results for the geometrical parameter $k=2$. For a given $\kappa$ obtained with $k=2, R_{A A}$ at $k=3$ decreases by $\sim 5-10 \%$ at $p_{T} \sim 10-20 \mathrm{GeV}$ and by $\sim 1-3 \%$ at $p_{T} \sim 50-100 \mathrm{GeV}$ as compared to $R_{A A}$ for $k=2$. The difference between $R_{A A}$ for $k=2$ and $k=3$ for the optimal values of $\kappa$ is very small (typically $\lesssim 1-2 \%$ ) at 


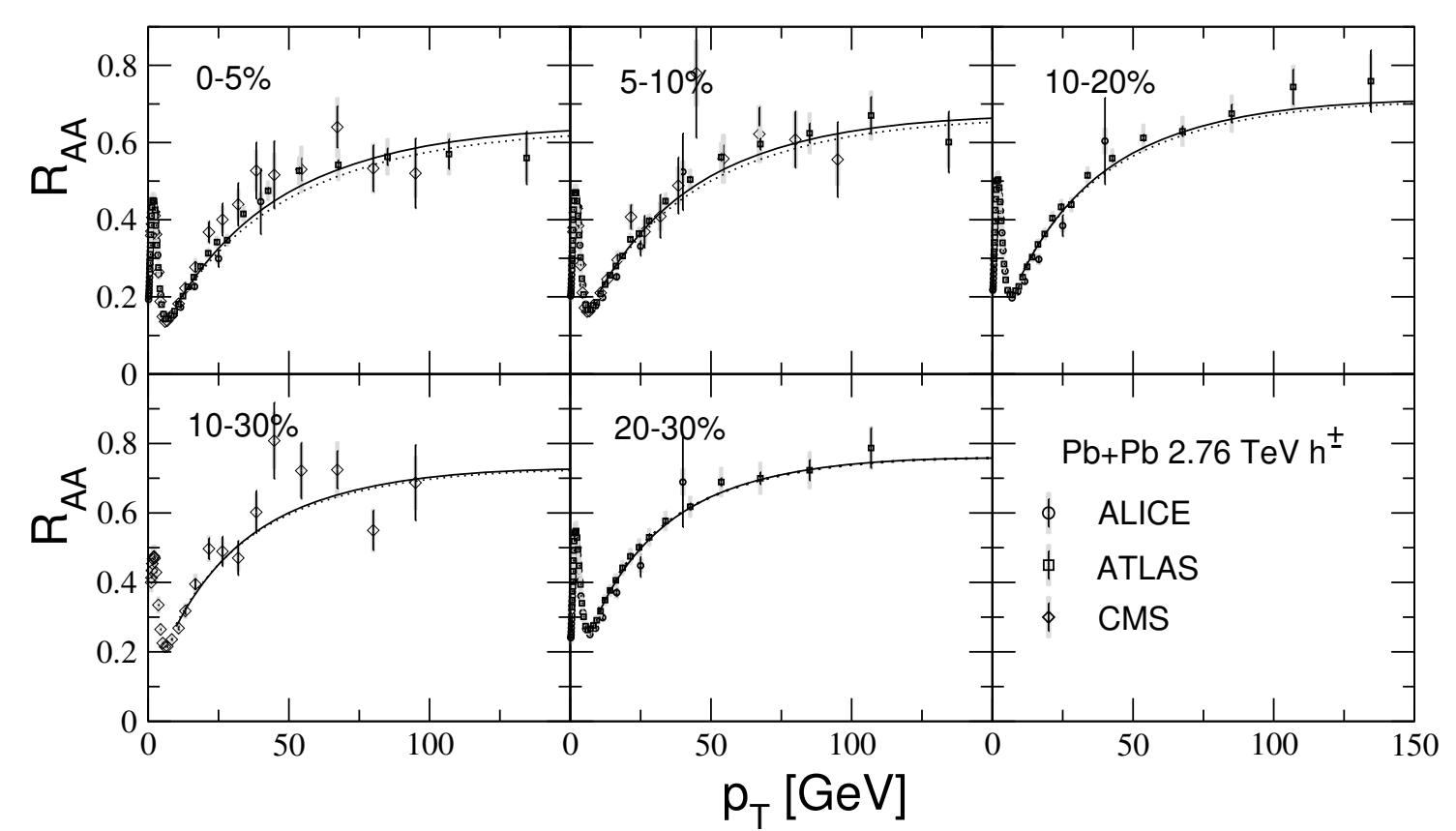

Figure 4. $R_{A A}$ of charged hadrons for $2.76 \mathrm{TeV} \mathrm{Pb}+\mathrm{Pb}$ collisions from our calculations with $k=2, c=0.8, \tau_{0}=0.5 \mathrm{fm}$ for scenarios without (solid) and with (dotted) mQGP formation in $p p$ collisions for the optimal parameters $\kappa=3.44$ and 2.57 obtained by fitting $R_{A A}$ in the range $10<p_{T}<120 \mathrm{GeV}$. Data points are from ALICE [75], ATLAS [76], and CMS [77].

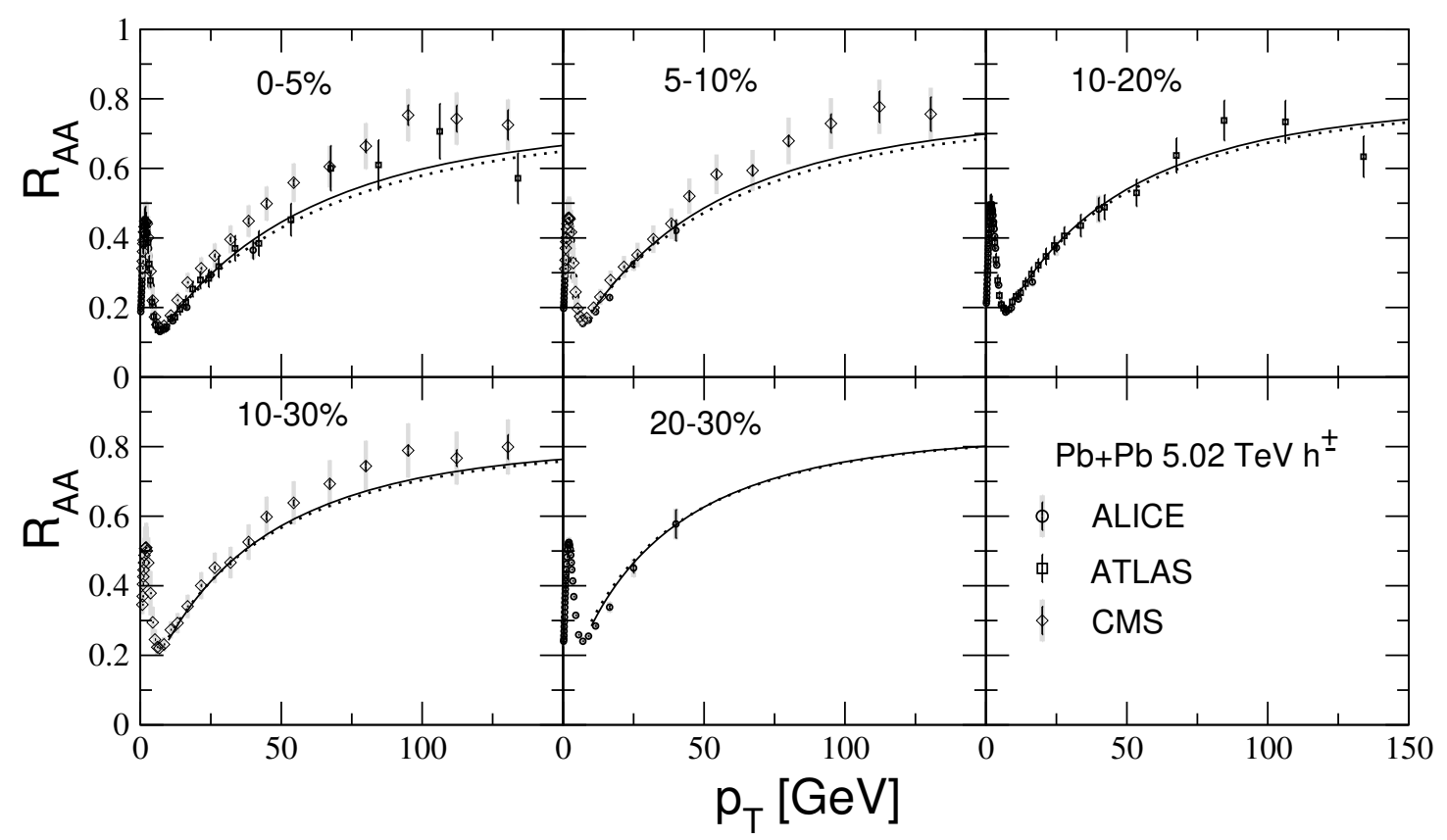

Figure 5. Same as in figure 4 for $\sqrt{s}=5.02 \mathrm{TeV}$ for the optimal parameters $\kappa=3.47$ and 2.5 . Data points are from ALICE [78], ATLAS [79], and CMS [80]. 


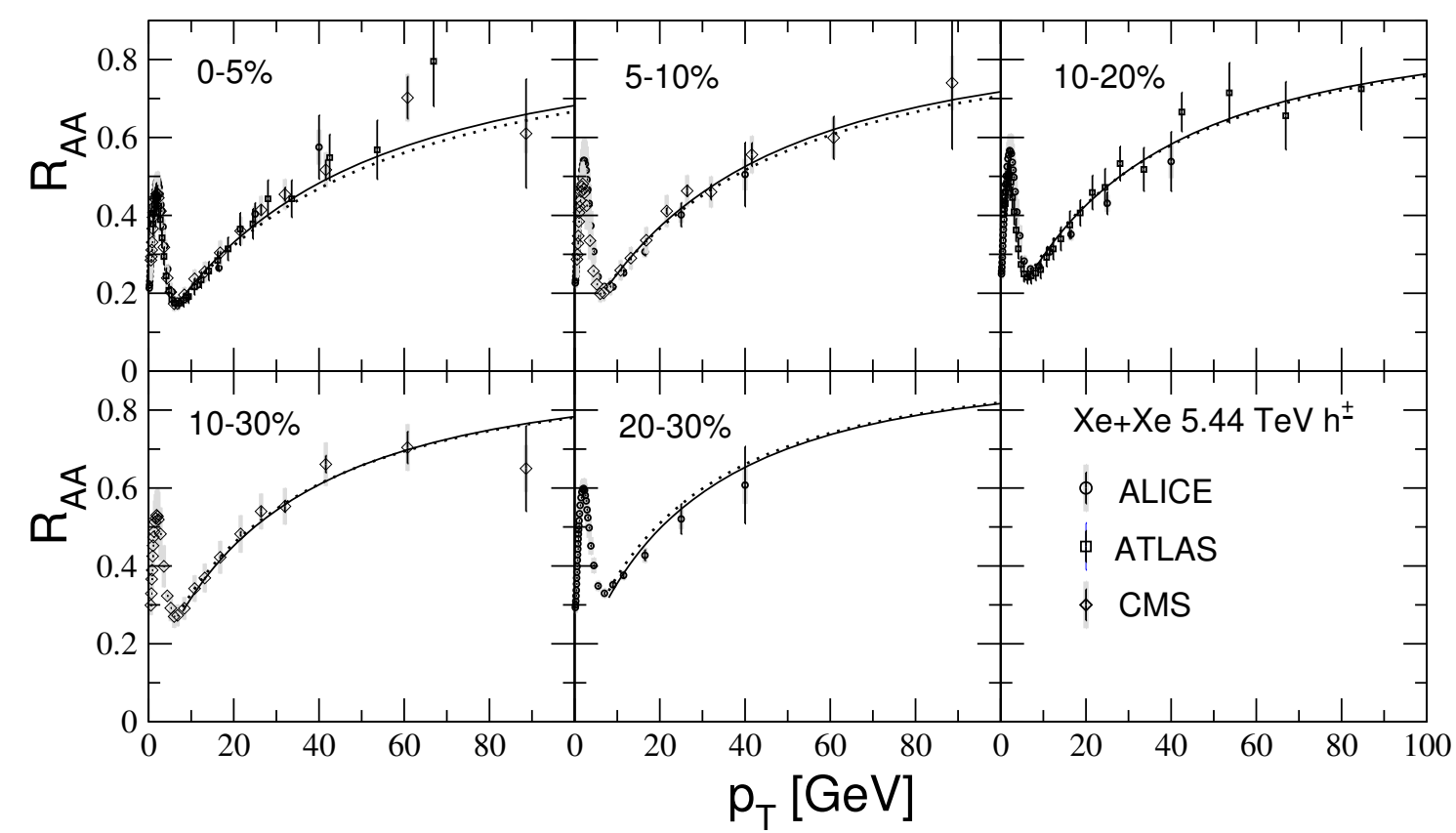

Figure 6. Same as in figure 4 for $5.44 \mathrm{TeV} \mathrm{Xe}+\mathrm{Xe}$ collisions for the optimal parameters $\kappa=3.59$ and 2.52. Data points are from ALICE [81], ATLAS [82], and CMS [83].

all $p_{T}$. Therefore we do not show the curves for $k=3$. From figures $4-6$ one can see that for optimal values of $\kappa$ the difference between theoretical $R_{A A}$ for our two scenarios is very small. From figures $4-6$ one can see that the theoretical curves for the scenarios with and without mQGP formation in $p p$ collisions agree quite well with experimental data. Our results for $R_{A A}$ obtained for $\tau_{0}=0.8 \mathrm{fm}$ (not shown) turn out to be very close to that for $\tau_{0}=0.5 \mathrm{fm}$. Even if $R_{A A}$ for $\tau_{0}=0.8 \mathrm{fm}$ is calculated with the optimal value of $\kappa$ obtained for $\tau_{0}=0.5 \mathrm{fm}$, it grows just by $\sim 1-2 \%$. This says that jet quenching in heavy ion collisions is not very sensitive to the initial stage of the fireball evolution. The results of our calculations for $c=0$ in the parametrization (2.5) are very close to that for $c=0.8$ (shown in figures 4-6), therefore we do not plot them as well.

In figure 7 we compare the results for $R_{A A}$ of $\pi^{0}$-mesons in $0.2 \mathrm{TeV} \mathrm{Au}+\mathrm{Au}$ collisions with the data from PHENIX [84]. The theoretical curves are obtained with the optimal values of $\kappa$ adjusted by fitting the LHC data. Note that a joint fit of the RHIC and the LHC data practically does not change the optimal values of $\kappa$ obtained from the LHC data, because the number of the data points for the LHC data set is much bigger than for the PHENIX data. From figure 7 one can see that the theoretical curves somewhat overshoot the data at $p_{T} \sim 10 \mathrm{GeV}$. However, the discrepancy between the results for RHIC and the LHC for the $T$-dependent $\alpha_{s}$ is much smaller than in the case of calculations with a unique, temperature independent, $\alpha_{s}$ [11]. Since conditions for formation of a mQGP in $p p$ collisions are less favorable than for the LHC energies, it is interesting to compare the RHIC data on $R_{A A}$ also for an intermediate scenario when mQGP formation in $p p$ collisions occurs only at the LHC energies. In this case the theoretical $R_{A A}$ for $0.2 \mathrm{TeV} \mathrm{Au}+\mathrm{Au}$ collisions should 


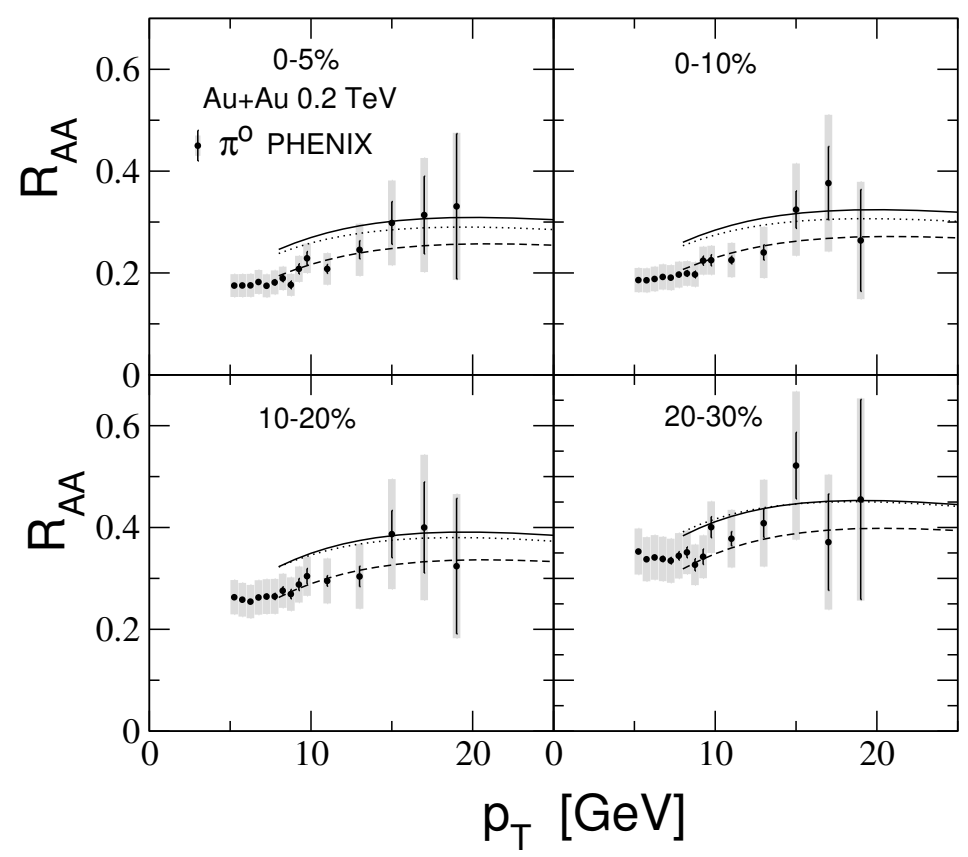

Figure 7. Same as in figure 4 for $R_{A A}$ of $\pi^{0}$ in $0.2 \mathrm{TeV} \mathrm{Au}+\mathrm{Au}$ collisions for the optimal parameters $\kappa=3.47$ and 2.55 determined by fitting all the $\mathrm{LHC}$ data on $R_{A A}$ in $\mathrm{Pb}+\mathrm{Pb}$ and $\mathrm{Xe}+\mathrm{Xe}$ collisions. The dashed curves show the results for $\kappa=2.55$, corresponding to the mixed scenario with mQGP formation in $p p$ collisions only at the LHC energies. Data points are from PHENIX [84].

be calculated without $1 / R_{p p}$ factor for the optimal $\kappa$ fixed in the scenario with mQGP formation from the LHC data on $R_{A A}$. In figure 7 we show the results for this case, using $\kappa=2.55$ obtained in the scenario with mQGP formation at the LHC energies. As one can see, it gives somewhat better agreement with the PHENIX data [84]. This fact supports the intermediate scenario without a fully developed hydrodynamic evolution of the QCD matter in $0.2 \mathrm{TeV} p p$ collisions. Of course, this interpretation of the discrepancy between $\alpha_{s}$ for the RHIC and the LHC energies is quite speculative, because our calculations include several simplifications, and the errors for the PHENIX data on $R_{A A}$ at $p_{T} \gtrsim 10 \mathrm{GeV}$ are rather large.

In figures 8 and 9 we compare the theoretical predictions for the flow coefficient $v_{2}$ with available experimental data for $2.76[85-87]$ and $5.02[88,89] \mathrm{TeV} \mathrm{Pb}+\mathrm{Pb}$ collisions. We show the theoretical results for the fireball azimuthal asymmetry $\epsilon_{2}$ obtained in the optical Glauber model and for the Monte-Carlo one (for the Gaussian entropy sources with width $\sigma=0.7 \mathrm{fm})$. The most significant difference in predictions of the optical and the MonteCarlo versions is for the most central (0-5\%) collisions. Although $v_{2}$ was not included into our $\chi^{2}$ analysis, the results for $v_{2}$ are in qualitative agreement with what is seen in the LHC data. Of course, one should bear in mind, that results for the azimuthal dependence are less robust. For instance, for $v_{2}$, which is sensitive to a delicate balance between jet modification in the directions along the short and long fireball semi-diameters, the errors due to the neglect of the transverse QGP motion and due to the use of a flat initial entropy distribution can potentially be larger than for the azimuthally averaged $R_{A A}$. Note that 


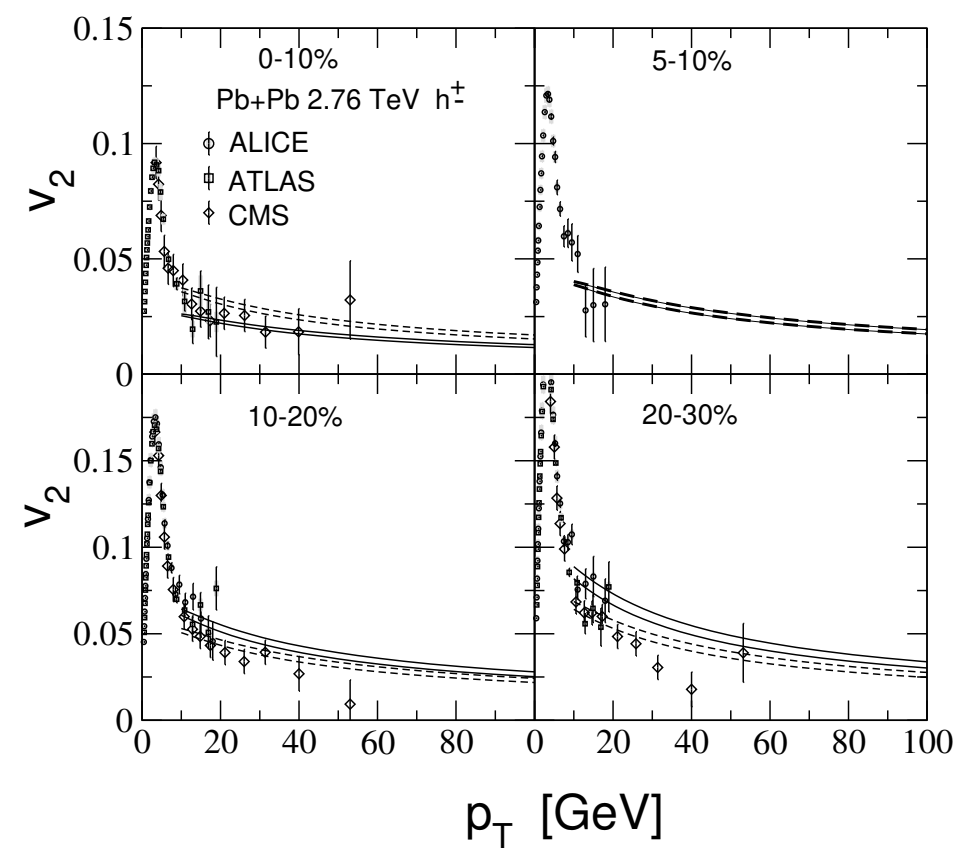

Figure 8. $v_{2}$ of charged hadrons in $2.76 \mathrm{TeV} \mathrm{Pb}+\mathrm{Pb}$ collisions for the initial fireball eccentricity $\epsilon_{2}$ calculated in the optical (solid) and Monte-Carlo (dashed) Glauber model for scenarios without and with (bottom to top) mQGP formation in $p p$ collisions. The parameters are the same as for figure 4. Data points are from ALICE [85], ATLAS [86], and CMS [87].

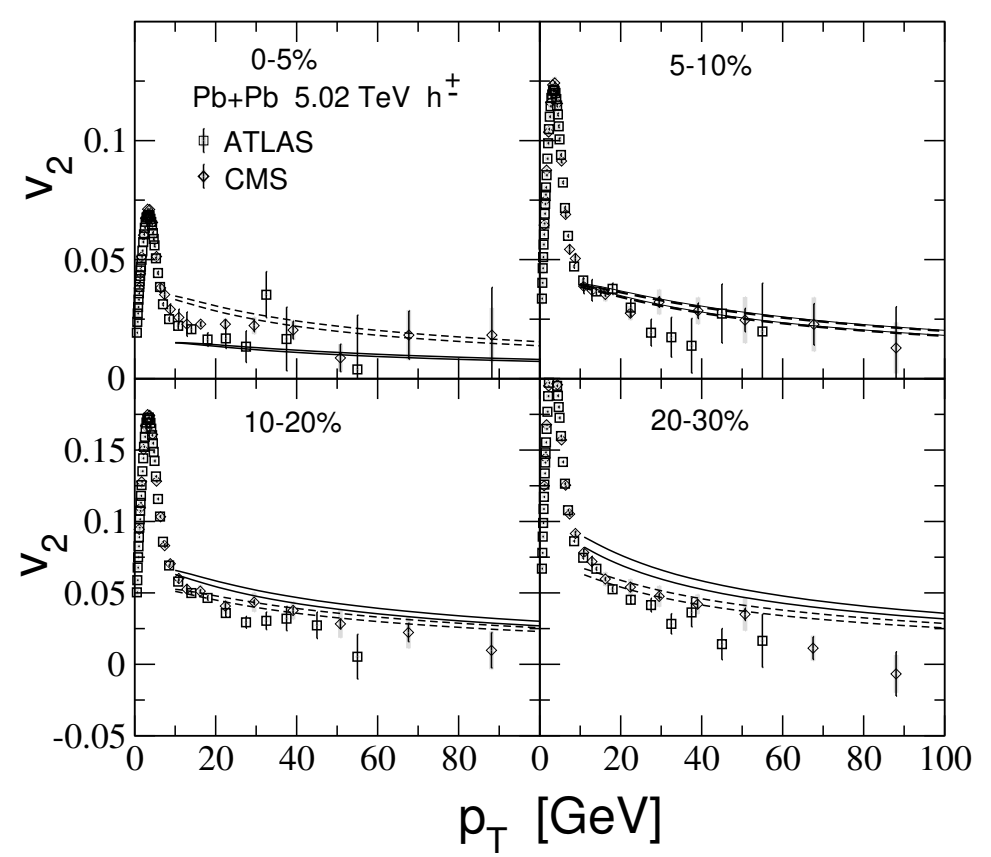

Figure 9. Same as in figure 8 for $\sqrt{s}=5.02 \mathrm{TeV}$. The parameters are the same as for figure 5 . Data points are from ATLAS [88] and CMS [89]. 


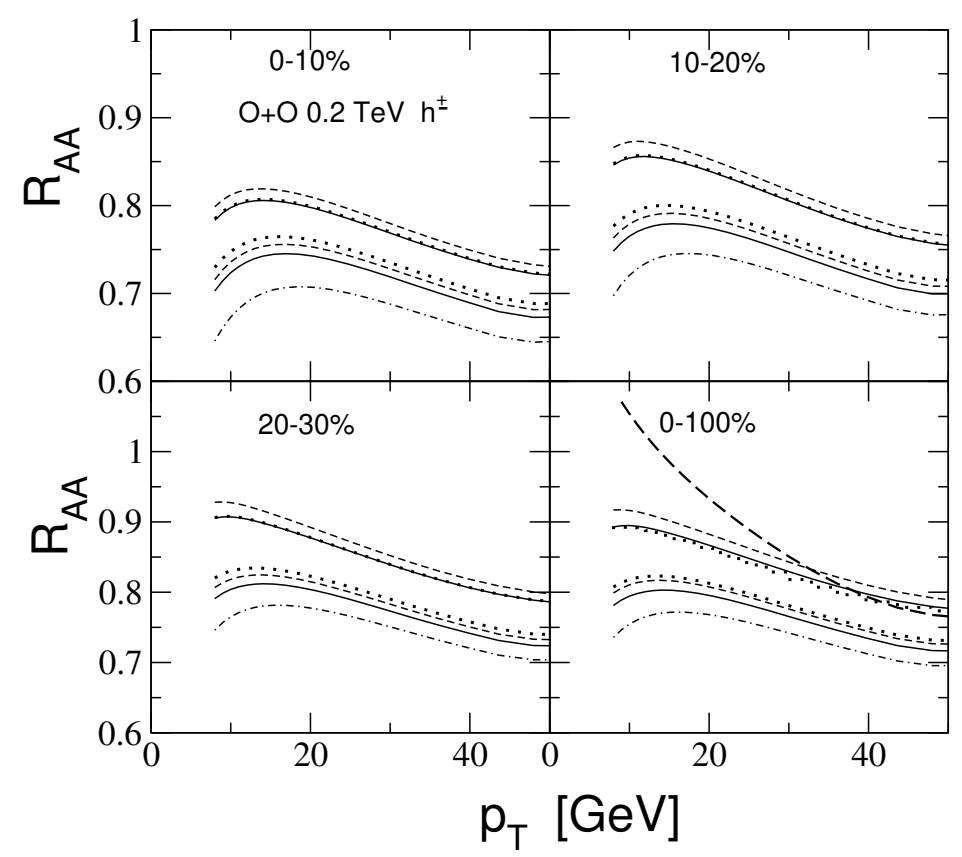

Figure 10. $R_{A A}$ of charged hadrons for $0.2 \mathrm{TeV} \mathrm{O}+\mathrm{O}$ collisions from our calculations for scenarios (top to bottom) with $(\kappa=2.55)$ and without $(\kappa=3.47)$ mQGP formation in $p p$ collisions. The solid and dashed curves are for $\tau_{0}=0.5$, and the dotted ones are for $0.8 \mathrm{fm}$. The solid and dotted curves are obtained for the geometrical parameter $k=2$, and the dashed curves are for $k=3$ (see text for explanation). The dash-dotted lines show the results (for $k=2, \tau_{0}=0.5 \mathrm{fm}, \kappa=2.55$ ) for the intermediate scenario when mQGP formation in $p p$ collisions occurs at the LHC energies, but it is absent at RHIC. The long dashed line shows $R_{A A}^{\mathrm{pdf}}$.

for $v_{2}$ the difference in the theoretical predictions for scenarios with and without mQGP is more pronounced than for $R_{A A}$. It is due to some enhancement of the strength of jet quenching for the scenario with mQGP formation (due to smaller value of $\kappa$ ) as compared to the one without mQGP. Contrary to the case of $R_{A A}$, for $v_{2}$ this difference is now not compensated by the presence of the $1 / R_{p p}$ factor, because this factor is immaterial for calculation of the azimuthal asymmetry.

Thus, we have established that the scenarios with and without mQGP can describe equally reasonably jet quenching in heavy ion collisions. Now, using the optimal values of $\kappa$, adjusted to fit $R_{A A}$ for heavy ion collisions, we can calculate $R_{A A}$ for $\mathrm{O}+\mathrm{O}$ collisions. In figures 10 and 11 we show the results for $R_{A A}$ in 0.2 and $7 \mathrm{TeV} \mathrm{O}+\mathrm{O}$ collisions for scenarios with and without mQGP formation in $p p$ collisions. We present results for three narrow centrality bins and for the minimum bias $R_{A A}$ (i.e., for $0-100 \%$ centrality interval). The latter quantity is particularly convenient for comparison with experiment, because for light ion collisions, due to large multiplicity fluctuations, the impact parameter becomes less correlated with centrality, which is defined experimentally via charged multiplicity. For $\sqrt{s}=0.2 \mathrm{TeV}$, as in figure 7 for $0.2 \mathrm{TeV} \mathrm{Au}+\mathrm{Au}$ collisions, we also show predictions for the intermediate scenario with mQGP formation in $p p$ collisions only at the LHC energies. As one can see, this scenario leads to the smallest $R_{A A}$ for $0.2 \mathrm{TeV} \mathrm{O}+\mathrm{O}$ collisions. To illustrate 


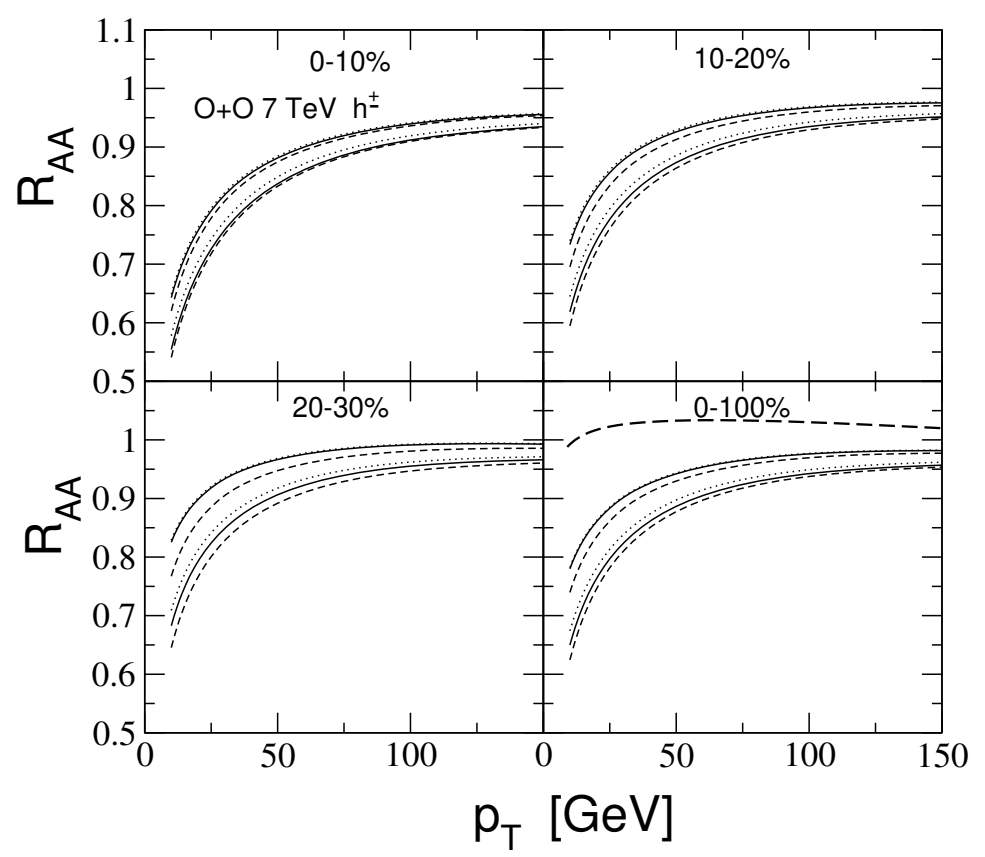

Figure 11. Same as in figure 10 (but now without dash-dotted lines) for $7 \mathrm{TeV} \mathrm{O}+\mathrm{O}$ collisions.

sensitivity of the results to $\tau_{0}$, in figures 10 and 11 we present the results for $\tau_{0}=0.5$ and $0.8 \mathrm{fm}$. As one can see, the difference between predictions for these two values of $\tau_{0}$ is rather small, especially for $\sqrt{s}=7 \mathrm{TeV}$. In figures 10 and 11, in addition to the real $R_{A A}$, we also plot the modification factor for hadron spectra only due to nuclear modification of the parton distribution functions for $A A$ collisions (which we denote $R_{A A}^{\mathrm{pdf}}$ ). For light ion collisions, when $R_{A A} \sim 1$, it is reasonable to characterize magnitude of jet quenching by $\Delta R_{A A}=R_{A A}^{\mathrm{pdf}}-R_{A A}$. From figures 10 and 11 we observe that, both for $\sqrt{s}=0.2$ and $7 \mathrm{TeV}$, there is a substantial difference in $\Delta R_{A A}$ for scenarios with and without mQGP formation in $p p$ collisions. Thus, although the discrepancy between $R_{A A}$ for these two scenarios are very small for heavy ion collisions, they give very different $\Delta R_{A A}$ for light ion collisions. To illustrate dependence of the theoretical predictions on the choice of the nuclear geometry parameter $k$, which we use to define the overlap region of the colliding nuclei, in figures 10 and 11, in addition to the version with $k=2$, we also plot the results for $k=3$. As one can see, there is no a significant difference between calculations for $k=2$ and 3. From figures 10 and 11 we can conclude that possible variation of $R_{A A}$ in $\mathrm{O}+\mathrm{O}$ collisions under change of the parameters $k$ and $\tau_{0}$ turns out to be smaller than the difference between predictions for our two scenarios without and with mQGP formation in $p p$ collisions.

From figure 11 one can see that for $7 \mathrm{TeV} \mathrm{O}+\mathrm{O}$ collisions the minimum bias $\Delta R_{A A}$ for scenarios without and with mQGP formation may differ by a factor of $\sim 1.5-2$ at $p_{T} \sim 50-150 \mathrm{GeV}$. Nevertheless, the $p_{T}$-dependence of $R_{A A}$ for both models are similar. Also, one should bear in mind that $R_{A A}^{\text {pdf }}$ has its own theoretical uncertainties. All this can make it difficult to discriminate between the scenarios without and with mQGP formation 


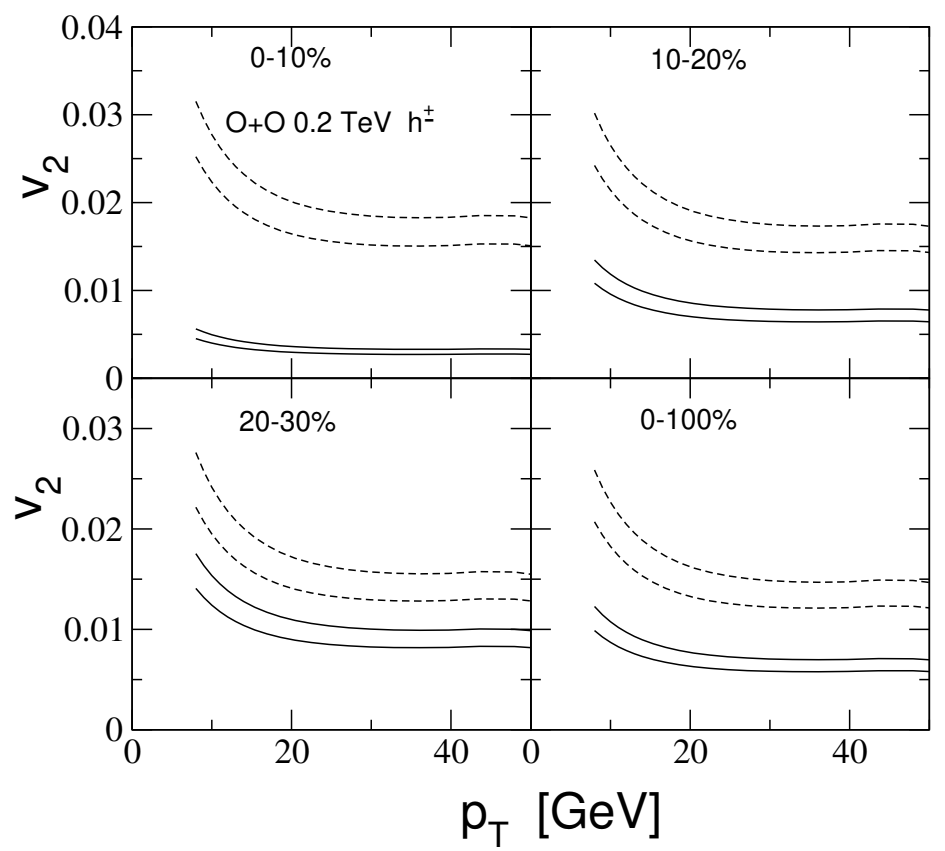

Figure 12. $v_{2}$ of charged hadrons in $0.2 \mathrm{TeV} \mathrm{O}+\mathrm{O}$ collisions for $\tau_{0}=0.5$ for the initial fireball eccentricity $\epsilon_{2}$ calculated in the optical (solid) and Monte-Carlo (dashed) Glauber model for scenarios without and with (bottom to top) mQGP formation in $p p$ collisions.

from comparison with the future LHC data. From figure 10 we observe that for $0.2 \mathrm{TeV}$ $\mathrm{O}+\mathrm{O}$ collisions the difference between scenarios with and without mQGP formation is somewhat more pronounced than at $\sqrt{s}=7 \mathrm{TeV}$. It is interesting that in this case the minimum bias $R_{A A}$ for the mQGP scenario becomes larger $R_{A A}^{\text {pdf }}$ at $p_{T} \gtrsim 35 \mathrm{GeV}$. An observation of such a crossover would support mQGP formation in $p p$ collisions.

In figures 12 and 13 we plot the results for the flow coefficient $v_{2}$ for the initial fireball azimuthal asymmetry $\epsilon_{2}$ obtained within the optical Glauber wounded nucleon model and within its Monte-Carlo counterpart. Similarly to $v_{2}$ for $\mathrm{Pb}+\mathrm{Pb}$ collisions, shown in figures 8 and 9 , we obtain somewhat larger $v_{2}$ for scenario with mQGP formation. As already said above, our results for $v_{2}$ in $\mathrm{O}+\mathrm{O}$ collisions are of illustrative character, because for $\mathrm{O}+\mathrm{O}$ collisions the effect of fluctuations of the initial entropy density is very important. For this reason our purpose is just to demonstrate the relation between the initial fireball ellipticity $\epsilon_{2}$ and $v_{2}$. From figure 13 we observe that for $7 \mathrm{TeV} \mathrm{O}+\mathrm{O}$ collisions $\epsilon_{2} / v_{2} \sim 20-25$ at $p_{T} \sim 50 \mathrm{GeV}$.

\subsection{Jet quenching in $p A$ collisions}

We have not touched yet jet quenching in $p A$ collisions. Similarly to formula (1.3), the theoretical observable nuclear modification factor for $p A$ collisions is given by $R_{p A}=R_{p A}^{\mathrm{st}} / R_{p p}$. Unfortunately, a conclusive comparison with experiment is presently impossible, because the available data on $R_{p A}$ are somewhat contradictory. ALICE measurement [78] gives the minimum bias $R_{p A}$ of charged hadrons in $5.02 \mathrm{TeV} p+\mathrm{Pb}$ collisions which is $\sim 0.92-1$ at $p_{T} \gtrsim 10$. More recent ALICE measurement [90] of $R_{p A}$ for $\pi^{0}$ mesons in $8.16 \mathrm{TeV} p+\mathrm{Pb}$ 


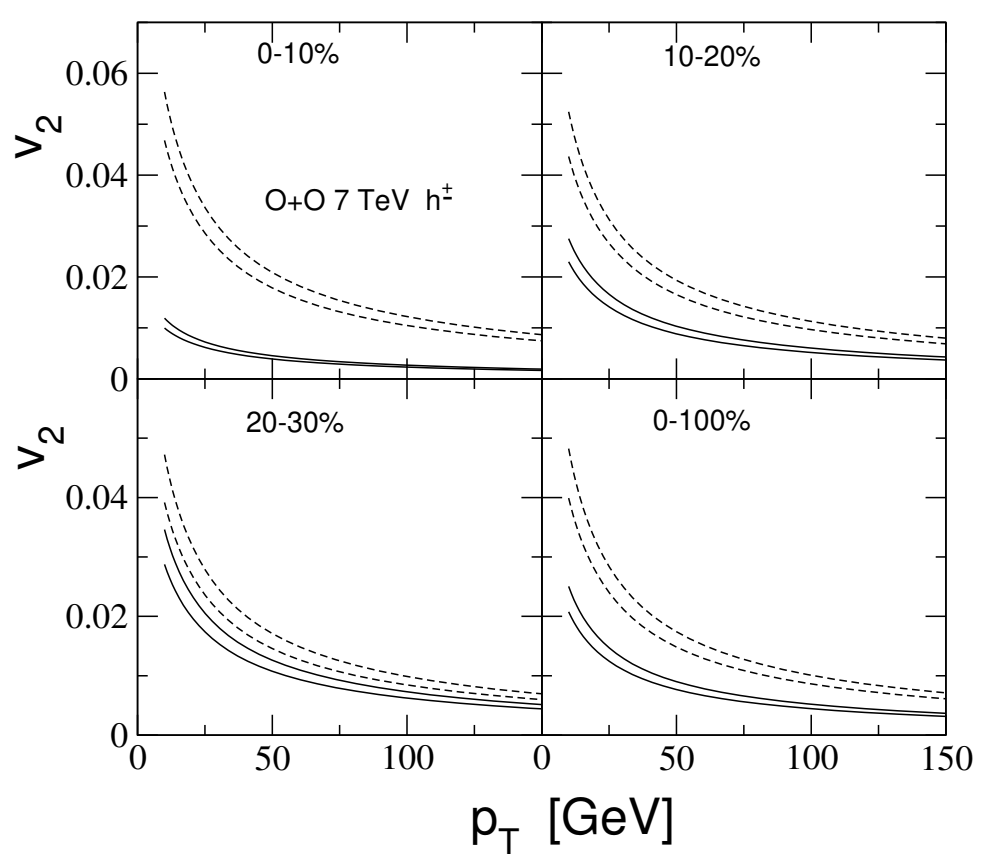

Figure 13. Same as in figure 12 for $\sqrt{s}=7 \mathrm{TeV}$.

collisions also gives $R_{p A}$ which is close to unity at $p_{T} \gtrsim 10 \mathrm{GeV}$. In contrast, the CMS collaboration [80] obtained for charged hadrons in $5.02 \mathrm{TeV} p+\mathrm{Pb}$ collisions $R_{p A} \sim 1.1-1.19$ at $p_{T} \gtrsim 10 \mathrm{GeV}$. The LHC data from ALICE and CMS seem to exclude the scenario when mQGP formation and jet quenching are absent in $p p$ collisions (i.e., $R_{p p} \approx 1$ ), but are present in $p A$ collisions (i.e., $R_{p A}<1$ ). If one assumes that the data from ALICE [78, 90] are correct, the question arises whether the scenario with mQGP formation, both in $p p$ and $p A$ collisions, can be consistent with $R_{p A}$ measured by ALICE. It is possible if the theoretical nuclear modification factor $R_{p A}^{\mathrm{st}}$ is just a little smaller than $R_{p p}$. Calculations of $R_{p A}^{\mathrm{st}}$ require detailed information on the QGP fireball in $p A$ collisions. We have performed preliminary calculations of $R_{p A}$ for 5.02 and $8.16 \mathrm{TeV} p+\mathrm{Pb}$ collisions using the fireball parameters obtained by modeling the UE fireball within the Monte-Carlo Glauber wounded nucleon model. The important difference between the minimum bias events and the jet events in the wounded nucleon Glauber picture is that in the jet events always there is one wounded $p N$ pair participating in the hard parton production, which produces the UE multiplicity density $d N_{\mathrm{ch}}^{\mathrm{ue}}(p p) / d \eta$ enhanced by the $K_{\mathrm{ue}} \sim 2.5$ factor, as compared to the minimum bias multiplicity density $d N_{\mathrm{ch}}^{\mathrm{mb}}(p p) / d \eta$. This leads to a two component structure of the fireball, with a high density core and the low density peripheral part (related to spectator wounded nucleons, that do not participate in the hard process). Our Monte-Carlo simulation shows that for $p+\mathrm{Pb}$ collisions the peripheral fireball part can give $\sim 20-30 \%$ of the total UE multiplicity density $d N_{\mathrm{ch}}^{\mathrm{ue}}(p A) / d \eta$. Since the entropy density in the peripheral part is rather small (the ideal gas temperature $\sim 150-170 \mathrm{MeV}$ ), one can expect that it should be close to the free-streaming regime, and its effect on jet quenching should be small. The core region of the UE fireball in 5.02(8.16) $\mathrm{TeV} p+\mathrm{Pb}$ collisions, which can 
have collective behavior, generates the charged multiplicity density $\sim 23 \pm 3(27 \pm 3)$. Calculations with such charged multiplicity densities give $R_{p A}=R_{p A}^{\mathrm{st}} / R_{p p}$ which agrees quite well with the ALICE data [78, 90]. Of course, this analysis is of preliminary character. It is highly desirable to investigate the jet quenching effect of the peripheral part of the fireball (that can be close to the free-streaming regime) and to perform calculations for a realistic fireball density profile accounting for the transverse flow (although we expect that the effect of the density variation and the flow corrections should be small (see section 2)). We leave this, more complicated, calculations for future work.

\section{Conclusions}

In the present work, we have studied jet quenching for heavy and light ion collisions within the LCPI approach [3] to induced gluon emission. Calculations of the induced gluon spectrum are performed with accurate treatment of the Coulomb effects, using the method suggested in [33]. We account for multiple gluon emission in the approximation of independent gluon radiation [39] in the form suggested in [32] (with some modifications made in [11]). We perform calculations for the temperature dependent running QCD coupling. We use parametrization of $\alpha_{s}(Q, T)$ which has a plateau around $Q=Q_{f r} \sim \kappa T$ (it is motivated by the lattice calculation of the effective QCD coupling in the QGP [41] and calculations within the functional renormalization group [42]).

We have investigated scenarios with and without mQGP formation in $p p$ collisions, and an intermediate scenario with mQGP formation only at the LHC energies. The parameter $\kappa$ has been fitted to the LHC data on the nuclear modification factor $R_{A A}$ in 2.76 and $5.02 \mathrm{TeV} \mathrm{Pb}+\mathrm{Pb}$, and $5.44 \mathrm{TeV} \mathrm{Xe}+\mathrm{Xe}$ collisions. We find that predictions of the models with and without mQGP formation in $p p$ collisions are very similar, and both of them lead to quite good description of the LHC heavy ion data on $R_{A A}$. The theoretical predictions for the flow coefficient $v_{2}$ are also in reasonable agreement with the LHC data. The optimal values of $\kappa$, fitted to the LHC data on $R_{A A}$, lead to reasonable description of the PHENIX data [84] on $R_{A A}$ in $0.2 \mathrm{TeV} \mathrm{Au}+\mathrm{Au}$ collisions in scenarios with and without mQGP formation in $p p$ collisions. Agreement with the PHENIX data becomes somewhat better for the intermediate scenario with mQGP formation in $p p$ collisions only at the LHC energies. Overall, our analysis shows that the experimental data on jet quenching in heavy ion collisions can equally well be described in scenarios without and with mQGP formation in $p p$ collisions.

Using the optimal values of the parameter $\kappa$, obtained by fitting $R_{A A}$ in heavy collisions, we perform calculations of $R_{A A}$ and $v_{2}$ for 0.2 and $7 \mathrm{TeV} \mathrm{O}+\mathrm{O}$ collisions which may be studied in future experiments at RHIC [15] and at the LHC [16-18]. We find that predictions of scenarios with and without mQGP formation for $R_{A A}-R_{A A}^{\mathrm{pdf}}$ begin to differ substantially for $\mathrm{O}+\mathrm{O}$ collisions. However, due to theoretical uncertainties for $R_{A A}^{\text {pdf }}$, and the fact that the $p_{T}$-dependence of $R_{A A}$ for both scenarios are similar, it may be difficult to discriminate between the scenarios without and with mQGP formation from comparison with the future LHC data. We find that for $0.2 \mathrm{TeV} \mathrm{O}+\mathrm{O}$ collisions the difference between scenarios with and without mQGP formation in $p p$ collisions is somewhat more 
pronounced. Our calculations show that in this case the minimum bias $R_{A A}$ for the mQGP scenario becomes larger $R_{A A}^{\text {pdf }}$ at $p_{T} \gtrsim 35 \mathrm{GeV}$.

For the medium modification factor $R_{p p}$ (that is not directly observable quantity) we obtain at $p_{T}=10 \mathrm{GeV} R_{p p} \sim 0.84-0.88$ at $\sqrt{s}=0.2 \mathrm{TeV}$, and $R_{p p} \sim 0.77-0.82$ at the LHC energies.

\section{Acknowledgments}

I am grateful to S. Tripathy for useful communication on some aspects of the recent ALICE results on jet quenching in small collision systems [31]. This work was performed under the Russian Science Foundation grant 20-12-00200 at Steklov Mathematical Institute.

Open Access. This article is distributed under the terms of the Creative Commons Attribution License (CC-BY 4.0), which permits any use, distribution and reproduction in any medium, provided the original author(s) and source are credited.

\section{References}

[1] M. Gyulassy and X.-n. Wang, Multiple collisions and induced gluon Bremsstrahlung in QCD, Nucl. Phys. B 420 (1994) 583 [nucl-th/9306003] [INSPIRE].

[2] R. Baier, Y.L. Dokshitzer, A.H. Mueller, S. Peigné and D. Schiff, Radiative energy loss of high-energy quarks and gluons in a finite volume quark-gluon plasma, Nucl. Phys. B 483 (1997) 291 [hep-ph/9607355] [INSPIRE].

[3] B.G. Zakharov, Fully quantum treatment of the Landau-Pomeranchuk-Migdal effect in QED and QCD, JETP Lett. 63 (1996) 952 [hep-ph/9607440] [INSPIRE].

[4] U.A. Wiedemann, Jet quenching versus jet enhancement: A Quantitative study of the BDMPS-Z gluon radiation spectrum, Nucl. Phys. A 690 (2001) 731 [hep-ph/0008241] [INSPIRE].

[5] M. Gyulassy, P. Lévai and I. Vitev, Reaction operator approach to nonAbelian energy loss, Nucl. Phys. B 594 (2001) 371 [nucl-th/0006010] [INSPIRE].

[6] P.B. Arnold, G.D. Moore and L.G. Yaffe, Photon and gluon emission in relativistic plasmas, JHEP 06 (2002) 030 [hep-ph/0204343] [INSPIRE].

[7] S. Shi, J. Liao and M. Gyulassy, Global constraints from RHIC and LHC on transport properties of QCD fluids in CUJET/CIBJET framework, Chin. Phys. C 43 (2019) 044101 [arXiv: 1808.05461] [INSPIRE].

[8] D. Zigic, I. Salom, J. Auvinen, M. Djordjevic and M. Djordjevic, DREENA-B framework: first predictions of $R_{A A}$ and $v_{2}$ within dynamical energy loss formalism in evolving $Q C D$ medium, Phys. Lett. B 791 (2019) 236 [arXiv:1805.04786] [InSPIRE].

[9] C. Andres, N. Armesto, H. Niemi, R. Paatelainen and C.A. Salgado, Jet quenching as a probe of the initial stages in heavy-ion collisions, Phys. Lett. B 803 (2020) 135318 [arXiv: 1902.03231] [INSPIRE].

[10] A. Huss, A. Kurkela, A. Mazeliauskas, R. Paatelainen, W. van der Schee and U.A. Wiedemann, Predicting parton energy loss in small collision systems, Phys. Rev. C 103 (2021) 054903 [arXiv:2007.13758] [INSPIRE]. 
[11] B.G. Zakharov, Updated analysis of jet quenching at RHIC and LHC within the light cone path integral approach, J. Phys. G 48 (2021) 055009 [arXiv:2007.09772] [INSPIRE].

[12] CMS collaboration, Observation of Long-Range Near-Side Angular Correlations in Proton-Proton Collisions at the LHC, JHEP 09 (2010) 091 [arXiv: 1009.4122] [INSPIRE].

[13] ATLAS collaboration, Observation of Long-Range Elliptic Azimuthal Anisotropies in $\sqrt{s}=13$ and $2.76 \mathrm{TeV}$ pp Collisions with the ATLAS Detector, Phys. Rev. Lett. 116 (2016) 172301 [arXiv: 1509.04776] [INSPIRE].

[14] K. Dusling and R. Venugopalan, Comparison of the color glass condensate to dihadron correlations in proton-proton and proton-nucleus collisions, Phys. Rev. D 87 (2013) 094034 [arXiv: 1302.7018] [INSPIRE].

[15] W. Li, Opportunities of $O O$ and $p O$ collisions at the LHC, LPCC Workshops, CERN, 4-10 February (2021) https://cds.cern.ch/record/2752019.

[16] Z. Citron et al., Report from Working Group 5: Future physics opportunities for high-density QCD at the LHC with heavy-ion and proton beams, CERN Yellow Rep. Monogr. 7 (2019) 1159 [arXiv: 1812.06772] [INSPIRE].

[17] R. Bruce, Opportunities of $O O$ and $p O$ collisions at the LHC, LPCC Workshops, CERN, 4 February (2021) https://cds.cern.ch/record/2751162.

[18] J. Brewer, A. Mazeliauskas and W. van der Schee, Opportunities of $O O$ and $p O$ collisions at the LHC, in Opportunities of $O O$ and $p O$ collisions at the LHC, (2021) [arXiv:2103.01939] [INSPIRE].

[19] R. Field, Min-Bias and the Underlying Event at the LHC, Acta Phys. Polon. B 42 (2011) 2631 [arXiv: 1110.5530] [INSPIRE].

[20] CDF collaboration, Charged Jet Evolution and the Underlying Event in p $\bar{p}$ Collisions at 1.8 TeV, Phys. Rev. D 65 (2002) 092002 [inSPIRE].

[21] PHENIX collaboration, Understanding the Role of Jet and Underlying Event in $p+p$ and $d+A u$ Collisions from PHENIX at RHIC, in 21st International Conference on Ultra-Relativistic Nucleus-Nucleus Collisions, (2009) [arXiv:0906.3776] [INSPIRE].

[22] ATLAS collaboration, Measurement of underlying event characteristics using charged particles in pp collisions at $\sqrt{s}=900 \mathrm{GeV}$ and $7 \mathrm{TeV}$ with the ATLAS detector, Phys. Rev. D 83 (2011) 112001 [arXiv: 1012.0791] [InSPIRE].

[23] CMS collaboration, Measurement of the Underlying Event Activity at the LHC with $\sqrt{s}=7 \mathrm{TeV}$ and Comparison with $\sqrt{s}=0.9 \mathrm{TeV}$, JHEP 09 (2011) 109 [arXiv:1107.0330] [INSPIRE].

[24] ALICE collaboration, Underlying Event measurements in $p p$ collisions at $\sqrt{s}=0.9$ and $7 \mathrm{TeV}$ with the ALICE experiment at the LHC, JHEP 07 (2012) 116 [arXiv:1112.2082] [INSPIRE].

[25] R. Campanini and G. Ferri, Experimental equation of state in proton-proton and proton-antiproton collisions and phase transition to quark gluon plasma, Phys. Lett. B $\mathbf{7 0 3}$ (2011) 237 [arXiv:1106.2008] [INSPIRE].

[26] M. Csanád, T. Csörgö, Z.-F. Jiang and C.-B. Yang, Initial energy density of $\sqrt{s}=7$ and $8 \mathrm{TeV} p+p$ collisions at the LHC, Universe 3 (2017) 9 [arXiv:1609.07176] [INSPIRE]. 
[27] ALICE collaboration, Enhanced production of multi-strange hadrons in high-multiplicity proton-proton collisions, Nature Phys. 13 (2017) 535 [arXiv:1606.07424] [INSPIRE].

[28] A. Kurkela, A. Mazeliauskas and R. Törnkvist, Collective flow in single-hit QCD kinetic theory, arXiv:2104.08179 [INSPIRE].

[29] B.G. Zakharov, Medium modification of photon-tagged and inclusive jets in high-multiplicity proton-proton collisions, Phys. Rev. Lett. 112 (2014) 032301 [arXiv:1307.3674] [INSPIRE].

[30] B.G. Zakharov, Parton energy loss in the mini quark-gluon plasma and jet quenching in proton-proton collisions, J. Phys. G 41 (2014) 075008 [arXiv:1311.1159] [InSPIRE].

[31] ALICE collaboration, Topological studies of charged particle production and search for jet quenching effects in small collision systems with ALICE, in 24th DAE-BRNS High Energy Physics Symposium, (2021) [arXiv:2103.07218] [INSPIRE].

[32] B.G. Zakharov, Jet quenching with running coupling including radiative and collisional energy losses, JETP Lett. 88 (2008) 781 [arXiv:0811.0445] [INSPIRE].

[33] B.G. Zakharov, Radiative parton energy loss and jet quenching in high-energy heavy-ion collisions, JETP Lett. 80 (2004) 617 [hep-ph/0410321] [INSPIRE].

[34] B.G. Zakharov, Jet quenching with T-dependent running coupling, JETP Lett. 112 (2020) 681 [arXiv: 2011.01526] [inSPIRE].

[35] B.G. Zakharov, Variation of jet quenching from RHIC to LHC and thermal suppression of QCD coupling constant, JETP Lett. 93 (2011) 683 [arXiv:1105.2028] [INSPIRE].

[36] B.G. Zakharov, Flavor dependence of jet quenching in pp collisions and its effect on $R_{A A}$ for heavy mesons, JETP Lett. 103 (2016) 363 [arXiv:1509.07020] [INSPIRE].

[37] B.A. Kniehl, G. Kramer and B. Potter, Fragmentation functions for pions, kaons, and protons at next-to-leading order, Nucl. Phys. B 582 (2000) 514 [hep-ph/0010289] [INSPIRE].

[38] T. Sjöstrand, L. Lönnblad, S. Mrenna and P.Z. Skands, PYTHIA 6.3 physics and manual, hep-ph/0308153 [INSPIRE].

[39] R. Baier, Y.L. Dokshitzer, A.H. Mueller and D. Schiff, Quenching of hadron spectra in media, JHEP 09 (2001) 033 [hep-ph/0106347] [INSPIRE].

[40] B.G. Zakharov, Medium-modification of photon-tagged jets in AA collisions, J. Exp. Theor. Phys. 125 (2017) 1071 [arXiv:1706. 03980] [INSPIRE].

[41] TUMQCD collaboration, Color screening in (2+1)-flavor QCD, Phys. Rev. D 98 (2018) 054511 [arXiv: 1804.10600] [INSPIRE].

[42] J. Braun and H. Gies, Running coupling at finite temperature and chiral symmetry restoration in QCD, Phys. Lett. B 645 (2007) 53 [hep-ph/0512085] [INSPIRE].

[43] A.C. Mattingly and P.M. Stevenson, Optimization of $R_{e^{+} e^{-}}$and 'freezing' of the $Q C D$ couplant at low-energies, Phys. Rev. D 49 (1994) 437 [hep-ph/9307266] [INSPIRE].

[44] Y.L. Dokshitzer, V.A. Khoze and S.I. Troian, Specific features of heavy quark production. LPHD approach to heavy particle spectra, Phys. Rev. D 53 (1996) 89 [hep-ph/9506425] [INSPIRE].

[45] O. Kaczmarek and F. Zantow, Static quark anti-quark interactions in zero and finite temperature QCD. I. Heavy quark free energies, running coupling and quarkonium binding, Phys. Rev. D 71 (2005) 114510 [hep-lat/0503017] [INSPIRE]. 
[46] P. Lévai and U.W. Heinz, Massive gluons and quarks and the equation of state obtained from SU(3) lattice QCD, Phys. Rev. C 57 (1998) 1879 [hep-ph/9710463] [INSPIRE].

[47] S. Borsányi et al., The QCD equation of state with dynamical quarks, JHEP 11 (2010) 077 [arXiv: 1007.2580] [INSPIRE].

[48] J.D. Bjorken, Highly Relativistic Nucleus-Nucleus Collisions: The Central Rapidity Region, Phys. Rev. D 27 (1983) 140 [inSPIRE].

[49] S. Kretzer, H.L. Lai, F.I. Olness and W.K. Tung, Cteq6 parton distributions with heavy quark mass effects, Phys. Rev. D 69 (2004) 114005 [hep-ph/0307022] [INSPIRE].

[50] K.J. Eskola, H. Paukkunen and C.A. Salgado, EPS09: A New Generation of NLO and LO Nuclear Parton Distribution Functions, JHEP 04 (2009) 065 [arXiv:0902.4154] [INSPIRE].

[51] R. Baier, D. Schiff and B.G. Zakharov, Energy loss in perturbative QCD, Ann. Rev. Nucl. Part. Sci. 50 (2000) 37 [hep-ph/0002198] [InSPIRE].

[52] B.G. Zakharov, Parton energy loss in an expanding quark-gluon plasma: Radiative versus collisional, JETP Lett. 86 (2007) 444 [arXiv:0708.0816] [INSPIRE].

[53] G.-Y. Qin, J. Ruppert, C. Gale, S. Jeon, G.D. Moore and M.G. Mustafa, Radiative and collisional jet energy loss in the quark-gluon plasma at RHIC, Phys. Rev. Lett. 100 (2008) 072301 [arXiv: 0710.0605] [INSPIRE].

[54] R. Baier, A.H. Mueller and D. Schiff, How does transverse (hydrodynamic) flow affect jet-broadening and jet-quenching?, Phys. Lett. B 649 (2007) 147 [nucl-th/0612068] [INSPIRE].

[55] B. Betz and M. Gyulassy, The effect of transverse flow on the nuclear modification factor at RHIC and LHC, AIP Conf. Proc. 1701 (2016) 060006 [InSPIRE].

[56] J.-Y. Ollitrault, Relativistic hydrodynamics for heavy-ion collisions, Eur. J. Phys. 29 (2008) 275 [arXiv: 0708.2433] [INSPIRE].

[57] B. Müller and K. Rajagopal, From entropy and jet quenching to deconfinement?, Eur. Phys. J. C 43 (2005) 15 [hep-ph/0502174] [INSPIRE].

[58] D. Kharzeev and M. Nardi, Hadron production in nuclear collisions at RHIC and high density QCD, Phys. Lett. B 507 (2001) 121 [nucl-th/0012025] [INSPIRE].

[59] B.G. Zakharov, Monte Carlo Glauber wounded nucleon model with meson cloud, J. Exp. Theor. Phys. 124 (2017) 860 [arXiv:1611.05825] [INSPIRE].

[60] B.G. Zakharov, Monte Carlo Glauber model with meson cloud: predictions for $5.44 \mathrm{TeV}$ Xe + Xe collisions, Eur. Phys. J. C 78 (2018) 427 [arXiv:1804.05405] [InSPIRE].

[61] STAR collaboration, Systematic Measurements of Identified Particle Spectra in pp, $d^{+} A u$ and Au+Au Collisions from STAR, Phys. Rev. C 79 (2009) 034909 [arXiv:0808.2041] [INSPIRE].

[62] ALICE collaboration, Centrality dependence of the charged-particle multiplicity density at mid-rapidity in Pb-Pb collisions at $\sqrt{s_{N N}}=2.76 \mathrm{TeV}$, Phys. Rev. Lett. 106 (2011) 032301 [arXiv: 1012.1657] [INSPIRE].

[63] ALICE collaboration, Centrality dependence of the charged-particle multiplicity density at midrapidity in $\mathrm{Pb}-\mathrm{Pb}$ collisions at $\sqrt{s_{\mathrm{NN}}}=5.02 \mathrm{TeV}$, Phys. Rev. Lett. 116 (2016) 222302 [arXiv: 1512.06104] [INSPIRE]. 
[64] ALICE collaboration, Centrality and pseudorapidity dependence of the charged-particle multiplicity density in Xe-Xe collisions at $\sqrt{s_{\mathrm{NN}}}=5.44$ TeV, Phys. Lett. B 790 (2019) 35 [arXiv: 1805.04432] [INSPIRE].

[65] B. Alver, M. Baker, C. Loizides and P. Steinberg, The PHOBOS Glauber Monte Carlo, arXiv:0805.4411 [INSPIRE].

[66] M. Rybczynski, G. Stefanek, W. Broniowski and P. Bozek, GLISSANDO 2: GLauber Initial-State Simulation AND mOre.., ver. 2, Comput. Phys. Commun. 185 (2014) 1759 [arXiv: 1310.5475] [INSPIRE].

[67] H.D. Vries, C.D. Jager and C.D. Vries, Nuclear charge-density-distribution parameters from elastic electron scattering, Atom. Data Nucl. Data Tabl. 36 (1987) 495.

[68] STAR collaboration, Pion interferometry in Au+Au collisions at $S(N N)^{* *}(1 / 2)=200-G e V$, Phys. Rev. C 71 (2005) 044906 [nucl-ex/0411036] [InSPIRE].

[69] ALICE collaboration, Centrality dependence of pion freeze-out radii in $\mathrm{Pb}$ - $\mathrm{Pb}$ collisions at $\sqrt{s_{\mathrm{NN}}}=2.76 \mathrm{TeV}$, Phys. Rev. C 93 (2016) 024905 [arXiv:1507.06842] [INSPIRE].

[70] R. Baier, Jet quenching, Nucl. Phys. A 715 (2003) 209 [hep-ph/0209038] [InSPIRE].

[71] A. Bzdak, B. Schenke, P. Tribedy and R. Venugopalan, Initial state geometry and the role of hydrodynamics in proton-proton, proton-nucleus and deuteron-nucleus collisions, Phys. Rev. C 87 (2013) 064906 [arXiv:1304.3403] [INSPIRE].

[72] B. Schenke, P. Tribedy and R. Venugopalan, Fluctuating Glasma initial conditions and flow in heavy ion collisions, Phys. Rev. Lett. 108 (2012) 252301 [arXiv:1202.6646] [INSPIRE].

[73] L. McLerran, M. Praszalowicz and B. Schenke, Transverse Momentum of Protons, Pions and Kaons in High Multiplicity pp and pA Collisions: Evidence for the Color Glass Condensate?, Nucl. Phys. A 916 (2013) 210 [arXiv:1306.2350] [INSPIRE].

[74] UA1 collaboration, A Study of the General Characteristics of pp Collisions at $\sqrt{s}=0.2-\mathrm{TeV}$ to $0.9 \mathrm{TeV}$, Nucl. Phys. B 335 (1990) 261 [INSPIRE].

[75] ALICE collaboration, Centrality Dependence of Charged Particle Production at Large Transverse Momentum in Pb-Pb Collisions at $\sqrt{s_{\mathrm{NN}}}=2.76 \mathrm{TeV}$, Phys. Lett. B $\mathbf{7 2 0}$ (2013) 52 [arXiv: 1208.2711] [INSPIRE].

[76] ATLAS collaboration, Measurement of charged-particle spectra in $\mathrm{Pb}+\mathrm{Pb}$ collisions at $\sqrt{s_{\mathrm{NN}}}=2.76 \mathrm{TeV}$ with the ATLAS detector at the LHC, JHEP 09 (2015) 050 [arXiv: 1504.04337] [INSPIRE].

[77] CMS collaboration, Study of high-pT charged particle suppression in PbPb compared to pp collisions at $\sqrt{s_{\mathrm{NN}}}=2.76 \mathrm{TeV}$, Eur. Phys. J. C 72 (2012) 1945 [arXiv:1202.2554] [INSPIRE].

[78] ALICE collaboration, Transverse momentum spectra and nuclear modification factors of charged particles in pp, $p$-Pb and Pb-Pb collisions at the LHC, JHEP 11 (2018) 013 [arXiv: 1802.09145] [INSPIRE].

[79] ATLAS collaboration, Measurement of nuclear modification factor $R_{\mathrm{AA}}$ in $\mathrm{Pb}+\mathrm{Pb}$ collisions at $\sqrt{s_{N N}}=5.02 \mathrm{TeV}$ with the ATLAS detector at the LHC, ATLAS-CONF-2017-012, CERN, Geneva (2017).

[80] CMS collaboration, Charged-particle nuclear modification factors in $\mathrm{PbPb}$ and $p \mathrm{~Pb}$ collisions at $\sqrt{s_{\mathrm{N} \mathrm{N}}}=5.02 \mathrm{TeV}$, JHEP 04 (2017) 039 [arXiv:1611.01664] [INSPIRE]. 
[81] ALICE collaboration, Transverse momentum spectra and nuclear modification factors of charged particles in Xe-Xe collisions at $\sqrt{s_{\mathrm{NN}}}=5.44$ TeV, Phys. Lett. B 788 (2019) 166 [arXiv: 1805.04399] [INSPIRE].

[82] ATLAS collaboration, Charged hadron spectra and dijet $p_{\mathrm{T}}$ correlations measured in $X e+X e$ collisions at $\sqrt{s_{N N}}=5.44 \mathrm{TeV}$ with the ATLAS detector, ATLAS-CONF-2018-007, CERN, Geneva (2018).

[83] CMS collaboration, Charged-particle nuclear modification factors in XeXe collisions at $\sqrt{s_{\mathrm{NN}}}=5.44 \mathrm{TeV}$, JHEP 10 (2018) 138 [arXiv:1809.00201] [INSPIRE].

[84] PHENIX collaboration, Neutral pion production with respect to centrality and reaction plane in Au+Au collisions at $\sqrt{s_{\mathrm{NN}}}=200 \mathrm{GeV}$, Phys. Rev. C 87 (2013) 034911 [arXiv: 1208.2254] [INSPIRE].

[85] ALICE collaboration, Anisotropic flow of charged hadrons, pions and (anti-)protons measured at high transverse momentum in Pb-Pb collisions at $\sqrt{s_{\mathrm{NN}}}=2.76 \mathrm{TeV}$, Phys. Lett. B 719 (2013) 18 [arXiv:1205.5761] [INSPIRE].

[86] ATLAS collaboration, Measurement of the pseudorapidity and transverse momentum dependence of the elliptic flow of charged particles in lead-lead collisions at $\sqrt{s_{\mathrm{NN}}}=2.76 \mathrm{TeV}$ with the ATLAS detector, Phys. Lett. B 707 (2012) 330 [arXiv:1108.6018] [INSPIRE].

[87] CMS collaboration, Azimuthal anisotropy of charged particles at high transverse momenta in $\mathrm{PbPb}$ collisions at $\sqrt{s_{\mathrm{NN}}}=2.76 \mathrm{TeV}$, Phys. Rev. Lett. 109 (2012) 022301 [arXiv:1204.1850] [INSPIRE].

[88] ATLAS collaboration, Measurement of the azimuthal anisotropy of charged particles produced in $\sqrt{s_{\mathrm{NN}}}=5.02 \mathrm{TeV} P b+P b$ collisions with the ATLAS detector, Eur. Phys. J. C 78 (2018) 997 [arXiv: 1808.03951] [INSPIRE].

[89] CMS collaboration, Azimuthal anisotropy of charged particles with transverse momentum up to $100 \mathrm{GeV} / \mathrm{c}$ in $\mathrm{PbPb}$ collisions at $\sqrt{s_{\mathrm{NN}}}=5.02 \mathrm{TeV}$, Phys. Lett. B 776 (2018) 195 [arXiv: 1702.00630] [INSPIRE].

[90] ALICE collaboration, Nuclear modification factor of light neutral-meson spectra up to high transverse momentum in $p$ - $P b$ collisions at $\sqrt{s_{\mathrm{NN}}}=8.16 \mathrm{TeV}$, arXiv:2104.03116 [INSPIRE]. 